San Jose State University

SJSU ScholarWorks

Master's Projects

Master's Theses and Graduate Research

Fall 2017

\title{
CACHE MANAGEMENT SCHEMES FOR USER EQUIPMENT CONTEXTS IN 5TH GENERATION CLOUD RADIO ACCESS NETWORKS
}

Gurpreet Kaur

San Jose State University

Follow this and additional works at: https://scholarworks.sjsu.edu/etd_projects

Part of the Computer Sciences Commons

\section{Recommended Citation}

Kaur, Gurpreet, "CACHE MANAGEMENT SCHEMES FOR USER EQUIPMENT CONTEXTS IN 5TH

GENERATION CLOUD RADIO ACCESS NETWORKS" (2017). Master's Projects. 563.

DOI: https://doi.org/10.31979/etd.vbfn-d72u

https://scholarworks.sjsu.edu/etd_projects/563

This Master's Project is brought to you for free and open access by the Master's Theses and Graduate Research at SJSU ScholarWorks. It has been accepted for inclusion in Master's Projects by an authorized administrator of SJSU ScholarWorks. For more information, please contact scholarworks@sjsu.edu. 


\title{
CACHE MANAGEMENT SCHEMES FOR USER EQUIPMENT CONTEXTS \\ IN $5^{\mathrm{TH}}$ GENERATION CLOUD RADIO ACCESS NETWORKS
}

\author{
A Writing Project \\ Presented to \\ The Faculty of the Department of Computer Science \\ San Jose State University \\ In Partial Fulfillment \\ Of the Requirements for the Degree \\ Master of Science in Computer Science
}

By

Gurpreet Kaur

December 2017 
(C) 2017

Gurpreet Kaur 


\section{ALL RIGHTS RESERVED}

The Designated Project Committee Approves the Project Titled

\section{CACHE MANAGEMENT SCHEMES FOR USER EQUIPMENT CONTEXTS IN $5^{\text {th }}$ GENERATION CLOUD RADIO ACCESS NETWORKS}

By

Gurpreet Kaur

APPROVED FOR THE DEPARTMENT OF COMPUTER SCIENCE SAN JOSE STATE UNIVERSITY

DECEMBER 2017
Dr. Melody Moh Department of Computer Science
Dr. Thomas Austin
Department of Computer Science
Dr. Robert Chun Department of Computer Science 


\section{ABSTRACT}

\section{By Gurpreet Kaur}

Advances in cellular network technology continue to develop to address increasing demands from the growing number of devices resulting from the Internet of Things, or IoT. IoT has brought forth countless new equipment competing for service on cellular networks. The latest in cellular technology is 5th Generation Cloud Radio Access Networks, or 5G C-RAN, which consists of an architectural design created specifically to meet novel and necessary requirements for better performance, reduced latency of service, and scalability. As part of this design is the inclusion of a virtual cache, there is a necessity for useful cache management schemes and protocols, which ultimately will provide users better performance on the cellular network. This paper explores a few different cache management schemes, and analyzes their performance in comparison to each other. They include a probability based scoring scheme for cache elements; a hierarchical, or tiered, approach aimed at separating the cache into different levels or sections; and enhancements to previously existing approaches including reverse random marking as well as a scheme based on an exponential decay model. These schemes aim to offer better hit ratios, reduced latency of request service, preferential treatment based on users' service levels and mobility, and a reduction in network traffic compared to other traditional and classic caching mechanisms. 


\section{TABLE OF CONTENTS}

LIST OF TABLES vii

LIST OF FIGURES viii

LIST OF ACRONYMS $\quad$ x

1. INTRODUCTION 1

2. BACKGROUND 3

2.1. Evolution of Cellular Network Technologies 3

2.2. 5G C-RAN 5

2.3. Cache Management 9

3. RELATED WORK 11

4. CACHE MANAGEMENT SCHEMES 14

4.1. Probability-Based Popularity Scoring 16

4.2. PBPS with Hierarchy 19

4.3. Reverse Random Marking 22

4.4. Reverse Random Marking with PBPS 23

4.5. EXD-AHP 26

4.6. Baseline Comparisons $\quad 30$

5. PERFORMANCE EVALUATION 32

5.1. Hit Rates 36

5.2. Miss Rates $\quad 40$

5.3. Latency 42

5.4. Cloud Writes 46 
6. CONCLUSION

7. REFERENCES 


\section{LIST OF TABLES}

Table 1. Example Analytical Hierarchical Process Matrix with Weights

Table 2. Simulated Algorithms

Table 3. Simulation Parameters and Values 


\section{LIST OF FIGURES}

Figure 1. Traditional Distributed RAN

Figure 2. Cloud-RAN $\quad 8$

Figure 3. Cache Management Flowchart 14

Figure 4. Visual Example of Tiered Cache $\quad 21$

Figure 5. Hit Rates with Cache Size $1250 \mathrm{MB}$

Figure 6. Hit Rates with Cache Size 2500 MB 37

Figure 7. Hit Rates with Cache Size $3750 \mathrm{MB}$

Figure 8. Hit Rates with Cache Size 5000 MB 39

Figure 9. Miss Rates with Cache Size 1250 MB 40

Figure 10. Miss Rates with Cache Size $2500 \mathrm{MB}$

Figure 11. Miss Rates with Cache Size 3750 MB 41

Figure 12. Miss Rates with Cache Size 5000 MB 42

Figure 13. Average Latency with Cache Size $1250 \mathrm{MB}$

Figure 14. Average Latency with Cache Size 2500 MB 44

Figure 15. Average Latency with Cache Size $3750 \mathrm{MB}$

Figure 16. Average Latency with Cache Size 5000 MB 45

Figure 17. Average Number of Cloud Writes with Cache Size 1250 MB 47

Figure 18. Average Number of Cloud Writes with Cache Size 2500 MB 47

Figure 19. Average Number of Cloud Writes with Cache Size 3750 MB 48

Figure 20. Average Number of Cloud Writes with Cache Size 5000 MB 49

Figure 21. Network Traffic with Cache Size $1250 \mathrm{MB}$ 
Figure 22. Network Traffic with Cache Size $1250 \mathrm{MB}$

Figure 23. Network Traffic with Cache Size $1250 \mathrm{MB}$

Figure 24. Network Traffic with Cache Size 1250 MB 


\section{LIST OF ACRONYMS}

BBU - Broadband Base Unit

CDMA - Code Division Multiple Access

C-RAN - Cloud Radio Access Network

EDGE - Enhanced Data Rates for Global System for Mobile Communication Evolution EPC - Evolved Packet Core

EXD-AHP - Exponential Decay with Analytical Hierarchical Process

GPRS - General Packet Radio Service

$\mathrm{HO}$ - Handover

IoT - Internet of Things

IP - Internet Protocol

LFU - Least Frequently Used

LTE - Long Term Evolution

LTE-A - Long Term Evolution Advanced

MME - Mobility Management Entity

OFDMA - Orthogonal Frequency Division Multiple Access

PBPS - Probability-Based Popularity Scoring

PCRF - Policy and Charging Rules Function

PGW - Packet Data Network Gateway

RAN - Radio Access Network

RRH - Remote Radio Head

RRM - Reverse Random Marking 
SGW - Serving Gateway

TDMA - Time Division Multiple Access

VM - Virtual Machine

VoIP - Voice Over Internet Protocol 


\section{INTRODUCTION}

Cellular networking technology has been advancing at impressive rates since its emergence in the technological market. With the onset of new devices, cellular networks have had to keep up with a growing demand for connectivity. This explosion of activity is evident by the Internet of Things, or IoT. IoT consists of more recently emerging devices that are not classic computers, including "smart" devices such as, for example, phones, interactive watches, televisions, and even household appliances such as thermostats. Technologies needing an Internet connection have even been projected to reach a staggering fifty billion devices within the next few years. IoT applications can, and will be, seen in developing areas such as smart grids, health services, homes, and even cities. IoT has had an incredible opportunity to flourish due to advances in physical sensors that can detect temperature and moisture, an increase in energy efficiency with better batteries, improvements in chip architecture resulting in smaller processing units, and also the adaptation of cloud computing. However, technological research is not the only thing aiding its growth. IoT is also expanding due to it being a lucrative field for business and consumerism.

With countless new equipment needing Internet access and constant connectivity, cellular networks have also advanced to be able to offer successful communication, speed of services, and scalability. The use of data centers has tremendously aided the future of cellular networks. Cloud data centers have allowed for the consolidation of computing resources on a massive scale, resulting in better utilization of both hardware and software 
resources as well as being able to enjoy the advantages of centralization, including virtualization, cost cutting, and uniform maintenance.

Since an important aspect of cellular networks is not just the support of massive numbers of users and devices, but also acceptable speeds of service, newer cellular architectures have included the presence of a cache [5]. Naturally, the cache must be efficiently maintained in order to make the best use of limited and available resources and offer users the quality of service they were promised.

This paper is organized as follows: The next section goes into brief detail about how cellular network services and architectures have evolved over time, as well as discuss the specifics about 5G C-RAN and the significance of cache management. Section III will cover related works on the topic of caching, followed by Section IV, which will dive into various cache management schemes and mechanisms. Section V will consist of a performance evaluation of those aforementioned schemes based on simulated data and results, and is followed by the conclusion in Section VI. 


\section{BACKGROUND}

\subsection{Evolution of Cellular Network Technologies}

Cellular networks have grown and developed impressively since the invention of the first mobile phone, Motorola's famous DynaTac 8000X. As generations progressed, users were provided with all manner of advances and service improvements. Over time, cellular networks attained capabilities for not just supporting mobile phones, but for millions of additional devices.

$1 \mathrm{G}$ introduced people to a new way of communication. Unlike ever before, users who could afford the early cellular phones could make calls while on the move. The architecture of these first generation networks was the simplest that would be seen, and telecommunication itself was analog based. Base stations would provide the subscribers, who were the customers of the cellular network, access to communication. The standards were based on the Advanced Mobile Phone System (AMPS), which was developed by Bell Labs in the 1980s. This technology utilized separate channels for each call that needed to be made, and therefore made use of Frequency Division Multiple Access (FDMA) [10]. Cells that were directly neighboring each other would operate on different frequencies in order to avoid any interference. The first generation of cellular networks was certainly a breakthrough, but had its fair share of limitations as well, including limited scalability due to an individual channel only being able to support a single user at any given particular time. In addition, analog transmissions used for voice communication were not ideally efficient in using the available frequency spectrum. 
The next generation, $2 \mathrm{G}$, introduced digital communication standards, replacing and upgrading the old analog signals and allowing for digital voice, which consisted of smaller packages that were compressed, as well as its encryption. Unlike in $1 \mathrm{G}$, this second generation started to also offer data to its customers, in the form of SMS messages, also known as texting. 2G technologies made use of Time Division Multiple Access [10]. The second generation was ultimately able to make better use of the available spectrum as it allowed support for more than one user over a single channel. $2 \mathrm{G}$ evolved into $2.5 \mathrm{G}$ and 2.75G, which respectively incorporated General Packet Radio Service (GPRS), which allowed for packet switching, and Enhanced Data Rates for GSM Evolution (EDGE), which allowed for better rates for transmitting data.

The third generation of telecommunication technology offered voice just as the previous generations had, but now offered high-speed data as well. Transfer rates were found to be between roughly $384 \mathrm{Kbps}$ and $2 \mathrm{Mbps}$, and were considered a substantial improvement. Code Division Multiple Access (CDMA) was used for multiplexing in 3G, which helped utilize the available signal spectrum to a much greater degree than seen before [10]. The third generation evolved to offer continuing higher speeds of data transfer rates in $3.5 \mathrm{G}$, and also later was able to offer Voice Over IP (VoIP) as well as utilize Orthogonal Frequency Division Multiple Access as part of 3.9G. The 3.9G technology also brought forth Long Term Evolution (LTE), which is a high speed standard specific to wireless telecommunications.

More recently, 4G has been offering data at very high speeds, ranging from 100 Mbps to as much as $1 \mathrm{Gbps}$. 4G offers increases in stability and speed, and has made 
enhancements to techniques using multiple antennas for communications. Channels were made wider with the user of OFDMA, and the core network has been more simplified and the architecture flattened [10]. Flat network architecture allows for the use of less equipment, therefore preserving resources. As an improvement to the prior generation, $4 \mathrm{G}$ offers Long Term Evolution Advanced (LTE-A). Compared to previous generations of cellular network technology, this fourth generation has offered a greater amount of data capacity to accommodate the growing number of devices craving Internet access. Furthermore, LTE-A is more stable than just LTE in terms of signal and data transfer.

Though strives have been made thus far with cellular networks, the ever increasing need for Internet access and that too at great speeds, in addition to the growth of the numbers of mobile devices, is what is fueling further improvements in this field. Furthermore, there are still many challenges, obstacles, and aspects in cellular networks that need to be addressed or can be enhanced [5]. As a result, the fifth generation of cellular networks is actively being researched, hoping to provide even better service yet.

\section{$2.25 G C-R A N$}

The fifth and newest generation ultimately aims to make substantial improvements over existing technologies. 5G will ideally have a peak data rate between 10 to $20 \mathrm{Gbps}$, the former being always offered to mobile devices and the latter under some very specific conditions set by the cellular providers. Latency of communication, in sending and receiving, should be decreased. $5 \mathrm{G}$ should offer connections to greater numbers of devices 
than previous generations, and should also have better handles on efficiency, including that of both energy consumption and spectral usage. In order to address these areas and make a number of these improvements, a more centralized architecture has been proposed for 5G, known as the Cloud Radio Access Network, or C-RAN [10, 18].

Before the proposal of Cloud-RAN, the radio access network was distributed, as seen below. The network would consist of User Equipment, or UE, which are the various devices and equipment belonging to mobile users that need access to the network, as well as multiple eNodeB.

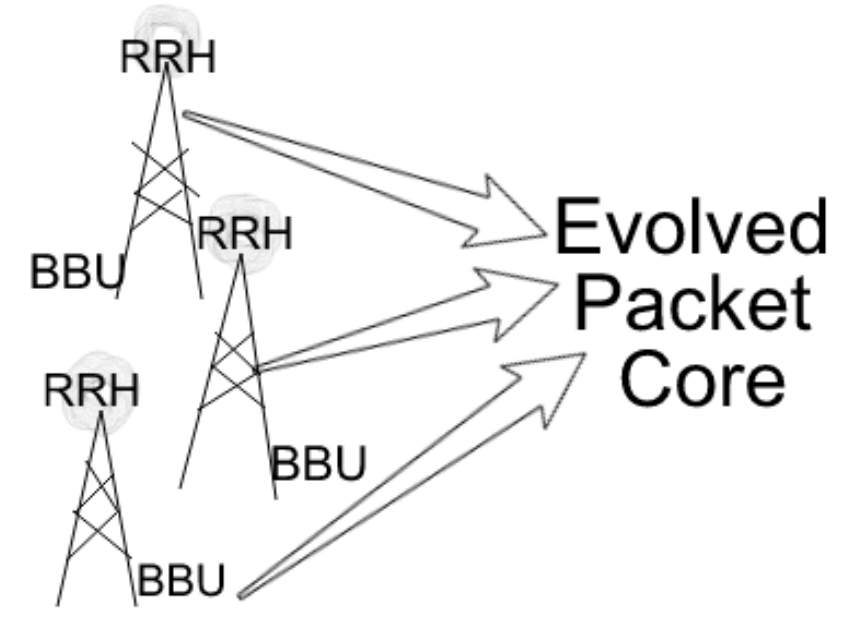

Figure 1. Traditional Distributed RAN

The remote radio heads (RRHs), which are essentially the cell towers, were each connected to their own Broadband Base Unit (BBU). The RRH and BBU together form the eNodeB. The radio heads are responsible for the transmission of signals, including both sending and receiving. The Broadband Base Units, on the other hand, have the job of converting the Internet Protocol (IP) packets into the digital signals that could then be used and interpreted. These broadband base units are additionally responsible for both the 
management and maintenance of the mobility of different users and their corresponding promised quality of services. The 5G and LTE-A architecture includes both the distributed Radio Access Network and its subsequent connection to the Evolved Packet Core (EPC). The EPC contains the fundamental framework for the network and how it operates, and includes a variety of components. Some of the main components are described briefly in the following. For instance, the Mobility Management Entity (MME) functions as the main node that is responsible for signaling. The MME plays an important role in the authentication of individual user devices on the network. Specifically, it tracks the user's location and will ultimately decide which gateway to access when a user registers with the network. Another component, called the Serving Gateway (SGW), takes instructions from the Mobility Management Entity on how to begin and end sessions with individual User Equipment. In addition, it aids in signaling between the MME and PGW. The Packet Data Network Gateway (PGW) can inspect and filter packets from users, in addition to the enforcement of data rates. Another element in the Evolved Packet Core is the Policy and Charging Rules Function (PCRF), which is responsible for managing and upholding the service rules. The PCRF can make decisions with user requests based on currently set policies, and is an important component in providing different Quality of Service (QoS) levels in the cellular network $[10,18]$.

In order to address some of the previously mentioned challenges present in $5^{\text {th }}$ generation cellular networks, a different type of architecture was proposed, which is Cloud-RAN, seen as follows $[5,18]$. 


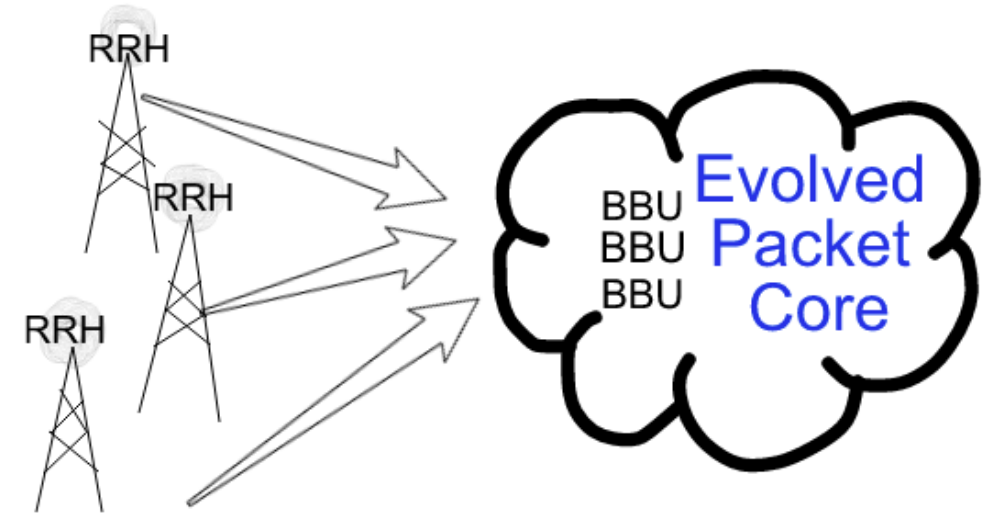

Figure 2. Cloud-RAN

There are some important and notable differences with this enhanced architecture. Firstly, C-RAN involves the radio heads being connected to a cloud data center. In the data center, the Broadband Base Units are pooled together. This pooling allows for a sharing of resources and provides the potential for better management of available resources, as centralization provides better control. In addition, the data center will consist of hosts, on which are running Virtual Machines (VMs). It is in these machines that the virtual base units are present. The virtualization of resources also aids in flexible programmability as well as lowered operational and maintenance costs compared to those of hardware resources [4]. Furthermore, the Cloud-RAN architecture is much better suited for scalability in the long run compared to its previous distributed counterpart. Lastly, the Cloud-RAN architecture is much better equipped to deal with handovers (HO). Handovers are basically when users are moving physically and need to connect or disconnect from the current tower and base unit and subsequently connect to a new radio head. There is a certain extent of the latency and performance compromises when this handover needs to occur in the previously distributed RAN architecture. After all, there is physical distance 
between the towers. Fortunately, in the cloud model, the Broadband Base Units are all pooled together and resources are shared. This way, handovers are handled in the same cloud data center, reducing the chance of users being dropped or going momentarily without service, and potentially reducing the time it takes to achieve connectivity when being mobile.

Next, it is important to understand the role of users in the network, and how the network is able to handle so many mobile devices at a time. Each user's device has a User Equipment Context (UEC). The UEC is what enables the unique identification of, for example, a particular phone or tablet. Each UEC will have an ID number to recognize its distinction from every other device. This context will also hold pertinent information about a user's session, and will have a record of what kind of subscription this user's device has as well as maintain the state of the device in relation to the session at the moment. A copy of all the UECs will be present in the physical storage of the cloud, but there must be a mechanism to provide quicker access to them. Because the information contained in the UEC must be present and up to date for all active users, and going to and from cloud storage takes extra time, a number of the UECs are also going to be stored in the virtual broadband base units as part of a cache.

\subsection{Cache Management}

Caching has been a useful tool in many applications and technologies, the latest of which is cellular networks. Since $5 \mathrm{G}$ aims to offer faster service than previous cellular 
generations, the use of a cache becomes altogether beneficial. Storing some number of User Equipment Contexts in the cache will allow them to be quickly accessed when needed, quickly read to obtain information, and quickly written to update the context. The delay that occurs in the cellular service will also be reduced, since caching will help prevent trips over the data center network to go back and forth from the physical storage in the cloud data center $[4,18]$.

Naturally, the management of cache resources becomes a very important aspect for the architecture of 5G C-RAN. Cache is a limited resource, so it must be used optimally. As a result, particular cache management schemes must be created in order to keep hit rates high, and subsequently miss rates, cloud write rates and latency low. These traits help define and implement the better service promises of 5G C-RAN. The following section will discuss related work in the context of fifth generation cellular networks, followed by a section explaining proposed, and enhancements of, caching schemes. 


\section{RELATED WORK}

Much research is constantly being done in handling a cache and improving its performance. For instance, Wang and Li [14] discussed ways of making cache both temporally and spatially aware by improving the organization of the cache design. Their design consisted of having two dynamically adjusting regions of cache, known as prefetch and victim regions. The purpose of the prefetch region is to take advantage of spatial locality by analyzing addresses, and the victim region would catch any elements evicted from higher in the memory stack. They combined these ideas with routing that was aware of bursts in the network, and were able to reduce the misses by up to a quarter. Their study brought to attention for this research the importance of having caching schemes that can adapt to changing workloads.

In another study, Ye et al. [16] aimed to ameliorate the performance of Solid State Drives (SSDs) by using a caching algorithm that would analyze the popularity of entire disk blocks and determine whether or not to replace an entire block. This scheme was able to reduce the number of times blocks were replaced, and could help in prolonging the longevity of the SSDs themselves. Relatedly, another study by Baek, Cho and Choi [2] involved caching for SSDs using probability models, specifically a geometric probability model, to determine which elements to place in the cache and which ones to evict. This study explained that good caching schemes do not have to be overly complex or computationally heavy, and can be based on simple mathematical models, as well as be optimized based on workload and either system or architecture specific parameters. Both 
these studies motivated this research by showcasing the usefulness of having a system to compare caching elements that looks beyond just primitive counters.

Floratou et al. [6] performed research for caching in the context of databases, specifically those of Big SQL and the Hadoop Distributed File System (HDFS). Elements in the cache would be given a score, which would be determined by some model, such as exponential decay, or simply assigned based on the number of accesses an element receives. The latter would be similar to caching done using the popular Least Frequently Used or Least Recently Used algorithms. The elements of the cache would consistently be ordered based on their scores, which would allow for eviction on one end of the list that contained the lowest scoring elements. Their algorithm was able to perform well in a variety of different types of workloads. The idea to score elements numerically motivated research behind the algorithms presented in this paper.

In a separate study about general caching, Zaidenberg, Gavish, and Meir [17] discussed novel caching algorithms that could potentially be applied to a variety of contexts and technologies. Amongst their multiple researched approaches included a Reverse Random Marking (RRM) algorithm, in which elements are flagged as unmarked when they enter the cache, and are flagged marked when they are accessed for the first time. Eviction is performed as a random selection out of the unmarked elements when needed. RRM is explained in more detail in the section discussing the caching algorithms, as well as its newly enhanced version. The authors also present a novel algorithm, in which the cache would be sectioned into a hierarchy of ordered lists. The popularly accessed elements would move up the hierarchy and unpopular elements would move 
down the hierarchy. The popularity of an element would be determined by the number of accesses that element receives, and the eviction of an element is done from the least popular end. The concept of using a hierarchy has been used in one of the algorithms presented in this paper, and has proved ultimately very useful in 5G C-RAN.

Huang, Zhao, and Zang [8] studied the effects on latency of a caching scheme specific to the traditional distributed Radio Access Network. As there are multiple eNodeB in that architecture, they researched and experimented with using a master and slave setup amongst the base stations. The master base station would help coordinate communication amongst the slave base stations, and the goal was to improve content delivery to users. If one eNodeB did not have content, the master base station could coordinate to get the content to the user from the appropriate node that had it, or as a last resort retrieve it from the cloud storage. Their experiments showed a decrease in the latency for accessing content. This study certainly brought forth the importance of maintaining good speeds of service.

Lastly, Anand and Barua [1] discussed the use of locking mechanisms in instruction cache in order to improve system performance, specifically that found in embedded systems. By locking particular lines of cache, they could assure certain elements remain in the cache for the duration of some system activity or event. This is especially useful when the most frequently and recently used items are locked and cannot be evicted, so hits are assured for those elements. 


\section{CACHE MANAGEMENT SCHEMES}

The general structure of how User Equipment Contexts can be handled in 5G Cloud-RAN can be represented by a flowchart adapted and adjusted from Tsai and Moh's research, illustrated in Figure 3.

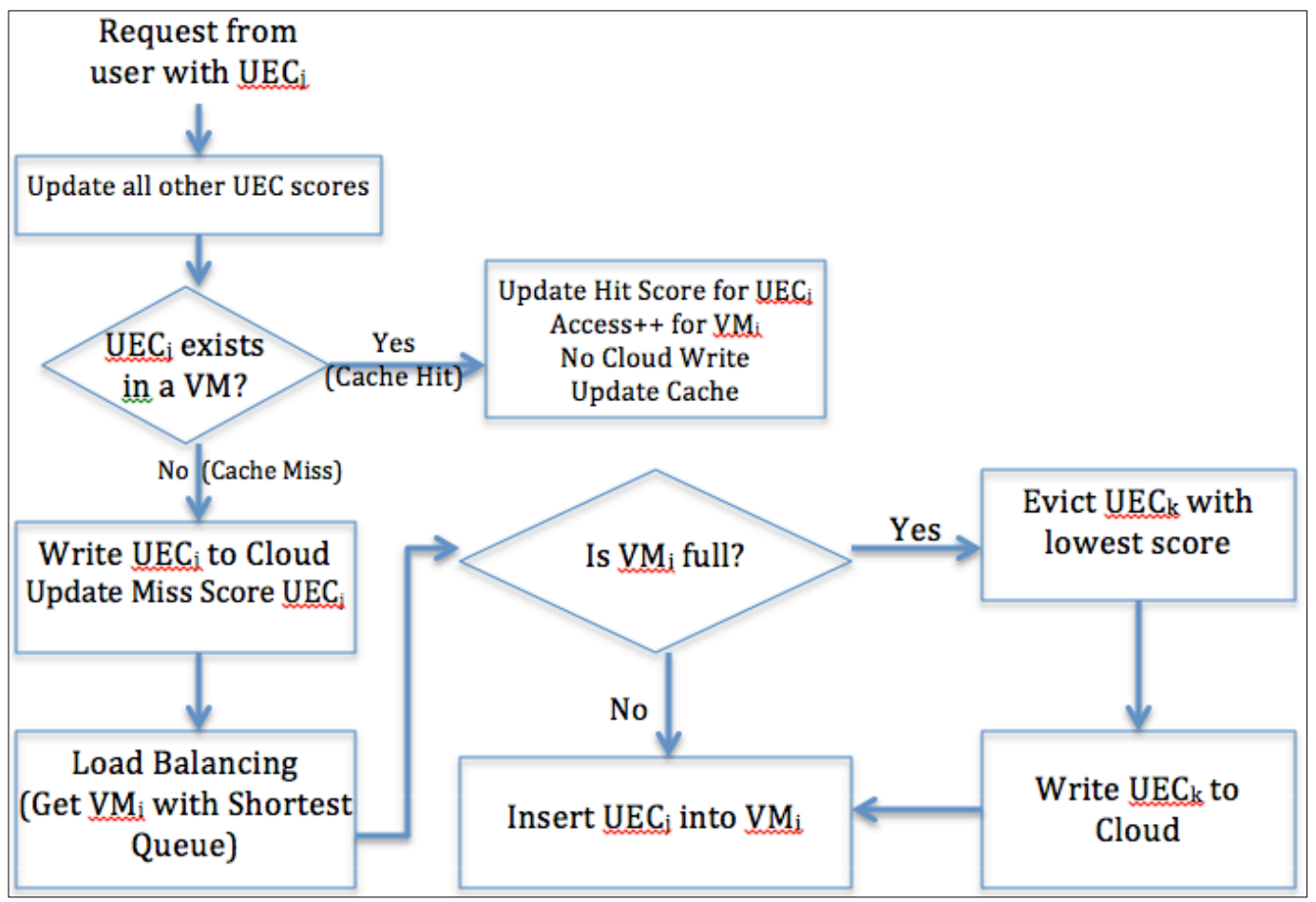

Figure 3. Cache Management Flowchart [13]

Requests from users are incoming, and each user is associated with a unique UEC. Once the UEC is identified, the information regarding all other UECs currently in the system cache are updated using a scoring process. However, note that the update of all other UECs can also happen near the end of this flow of events. If the currently incoming UEC is present in one of the virtual machines in the data center, the UEC is simply updated in the cache, and no cloud write is required. This is a scenario that is considered a cache hit. 
On the other hand, if the UEC is not present in one of the virtual machines, meaning there is a cache miss, it must be written to the cloud, and a load balancing algorithm must be performed in order to determine in which virtual machine the UEC is to be placed. The algorithm chosen to load balance involves finding the virtual machine that has the shortest queue of elements waiting to be processed. This particular method was chosen based on a study which determined the shortest queue approach was the most preferred for load balancing in 5G C-RAN due to its ability to offer a significantly even distribution of incoming requests with reasonable computational effort in comparison to other load balancing methodologies $[4,5]$. Once the virtual machine is successfully chosen, a check is performed to determine whether or not that virtual machine has room to accommodate an additional UEC. If the virtual machine has room, meaning the cache is not full, the UEC is simply inserted into the chosen virtual machine's cache. If the virtual machine is full, then the cache management scheme will choose a UEC to evict, typically one with the lowest score. Once the eviction is performed, the evicted UEC must be written to the cloud and the previously incoming UEC can be inserted into the cache.

In this general structure, there is much room for how exactly to handle the UECs, specifically how to handle insertion, eviction, and writes. This is where the various cache management schemes come into play. The succeeding schemes all present and discuss different ways of managing the UEC elements in the context of the cache. 


\subsection{Probability-Based Popularity Scoring (PBPS)}

As mentioned in Baek, Cho, and Choi's research, probability based models and functions can be used to aid in effective cache management [2]. Consider, for instance, the geometric probability model as follows:

$$
\mathrm{y}=\mathrm{p}(1-\mathrm{p})^{\mathrm{k}}
$$

This function will determine the probability of an event occurring based on success factor "p" and fail factor "k." A function like this can potentially be very useful in cache management for a few reasons.

Firstly, success in the context of caching can be defined as getting a hit in the cache. Likewise, failure can be considered experiencing a cache miss. The above function can be altered and adjusted to take into consideration hit and miss ratios to determine the popularity of a particular element. The hit ratio, which is calculated as a score, representing the hits a particular UEC has received in the cache based on its service level, divided by the total number of requests so far, can take the place of "p." Similarly, the miss ratio, which is the score representing the misses a particular UEC experiences divided by the total number of requests, can take the place of "k." By changing the above function to adapt to a cache management scheme for $5 \mathrm{G}$, a new function that is proposed and can be used for handling and scoring each UEC becomes the following:

$$
\frac{\text { hit score based on } L_{-} i}{\# \text { requests }}\left(1-\left(\frac{\text { hit score based on } L_{-} i}{\# \text { requests }}\right)\right)^{\frac{\text { miss score }}{\# \text { requests }}}
$$

This function takes into consideration both the hits and misses for each UEC at any given point in the cache management scheme, and evaluates to an overall score. The overall 
score of a UEC represents how popular the UEC is based on its hits and misses. A greater score of a UEC indicates a greater popularity of the UEC. Because the number of requests is constantly changing, this function works by dynamically scoring UECs in the system, therefore providing justice to both frequency and recency. This equation no longer evaluates to a strict probability, since the definitions of success and failure have been altered. However, since it is probability based, the overall scores evaluate to numbers between 0 and 1 inclusive. Furthermore, the hit and miss scores seen in the equation can be calculated depending on the quality of service promised to a particular user. This system can use a point reward for hits, in which the quantity of the reward is dependent on service level. As an example, a UEC in a better service level would have the hit score updated by a larger reward amount than a UEC in a lower service level that also experienced a hit. This is how differentiations are made between service levels and preferential treatment provided to better service quality subscriptions. The exact quantity of reward given based on service level can vary and be adjusted based on individual system needs. Furthermore, due to the structure of this scoring equation, the hit and miss scores for all UECs can be initialized to 1 by default, to prevent excess recurring scores of 0. Initializing with 1 will assure that scores are always calculated to nonzero values, allowing for the accurate and fair comparison of popularity and the avoidance of multiple 0 values that would complicate eviction. The following are the steps taken for this Probability-Based Popularity Scoring scheme:

\section{Probability-Based Popularity Scoring Algorithm}

1. For each user request $\mathrm{UEC}_{\mathrm{j}}$ with $\mathrm{L}_{\mathrm{i}}$ 
2. If $\mathrm{UEC}_{\mathrm{j}}$ is present in one of the VMs (cache hit)

3. Update hit score of $\mathrm{UEC}_{\mathrm{j}}$ based on service level and calculate new overall score using equation (2)

4. Update content of $\mathrm{UEC}_{\mathrm{j}}$ in the cache

5. Return;

6. Else if $\mathrm{UEC}_{\mathrm{j}}$ is not present in one of the VMs (cache miss)

7. Update miss score of $\mathrm{UEC}_{\mathrm{j}}$ and calculate new overall score using equation (2)

8. Write $\mathrm{UEC}_{\mathrm{j}}$ to the cloud

9. Load balance: select $\mathrm{VM}_{\mathrm{i}}$ with the shortest queue

10. If the $\mathrm{VM}_{\mathrm{i}}$ has space for the current $\mathrm{UEC}_{\mathrm{j}}$

11. Insert $\mathrm{UEC}_{\mathrm{j}}$ into the $\mathrm{VM}_{\mathrm{i}}$ 's cache

12. Return;

13. Else if the $\mathrm{VM}_{\mathrm{i}}$ is full

14. Find $\mathrm{UEC}_{\mathrm{k}}$ which has the lowest score

15. Evict $\mathrm{UEC}_{\mathrm{k}}$ from the $\mathrm{VM}_{\mathrm{i}}$ 's cache

16. Write $\mathrm{UEC}_{\mathrm{k}}$ to the cloud

17. Insert $\mathrm{UEC}_{\mathrm{i}}$ into the $\mathrm{VM}_{\mathrm{i}}$ 's cache

18. Return;

19. Update all other UECs in the cache using equation (2) with total number request parameter updated

This algorithm presents multiple advantages that are extremely useful in this $5 \mathrm{G}$ setting. As previously mentioned, the reward system for hits differentiates service levels. 
Also, since the equation factors in the total number of requests that have come through the network, it tackles a problem that is persistent in primitive algorithms such as ones like LFU or LRU. For example, in LFU, an element in the cache that was very popular over some time will have had many accesses and will remain in the cache for a very long period of time. Even if the item is no longer accessed or popular, it stays in the cache because at some point in the past it did receive very many accesses. However, items which are no longer popular, even if they once were, should not be kept sitting idle and taking up space in the limited resource that is the cache. In the PBPS function, the total number of requests is always an increasing parameter in the equation. This way, if a once-popular element is sitting in the cache and no longer being accessed, its overall score will steadily be decreasing, and it will be in a better position for eviction. PBPS therefore is able to dynamically account for changes in user requests and workloads.

\subsection{Probability-Based Popularity Scoring (PBPS) with Hierarchy}

The previously mentioned caching scheme handles the UECs and the cache well in that it dynamically adjusts scores for all the UECs in the system, and also makes differentiations based on service levels. However, the Probability-Based Popularity Scoring scheme can be further adjusted and aided with the sheer setup and calculated organization of the cache resources. Though PBPS differentiates between service levels by updating hit scores using different numbers of rewards for different quality of services, there is a question of fairness in service as those rewards are arbitrary. In the worst case 
for PBPS, better service levels can be overwhelmingly preferred for remaining in the cache while lower service levels overwhelmingly are facing eviction from the cache. This is a form of extreme behavior that would result in a great hit rate for the best service level, and a catastrophic hit rate for lower service levels. Because of the presence of this worstcase scenario, another enhanced approach to caching is proposed, which consists of the cache itself being separated into hierarchies or tiers.

The cache can be separated into particular sections, in which case each service level would have a section of the cache. This would form a type of hierarchy, in which particular User Equipment Contexts can only be added to or evicted from the section of cache that is dedicated to the service level to which they belong. In order to maintain the preferential treatment of better service levels, a larger amount of the cache can be dedicated to better service levels.

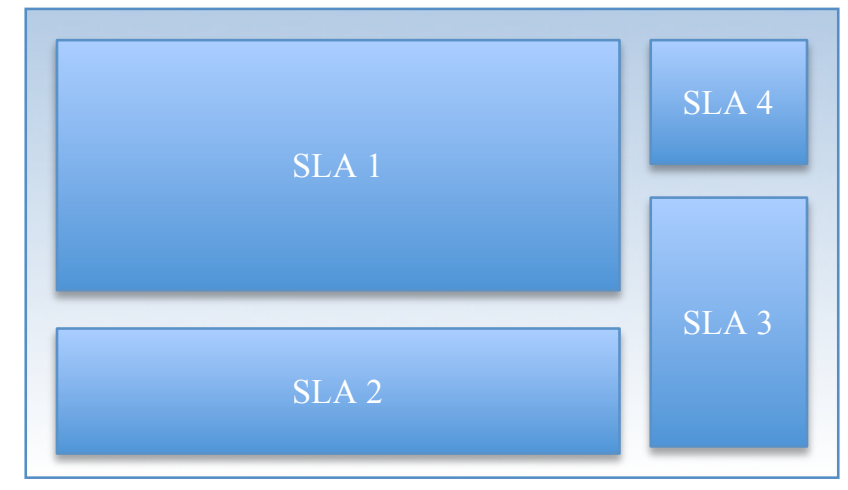

Figure 4. Visual Example of Tiered Cache

As an example, consider the case in which there is an average quality of service, and a superior quality of service. A caching separation and hierarchy could be maintained by dedicating, say, $60 \%$ of the cache to the superior service elements, and $40 \%$ of the cache 
to the average service elements. The exact separation of the cache into a hierarchy will depend on the number of service levels, and to what extent the provider wants to give preferential treatment to higher service levels over lower service levels. In any case, the probability-based popularity scoring scheme previously mentioned can still stay in place, and User Equipment Contexts can be continued to be given scores based on growing numbers of hits, misses, and requests. With the addition of the hierarchy, cache management will be done in a section of the cache, instead of the entire cache, based on what service level the incoming UEC has. The scoring scheme will still help in determining keeping the higher scoring elements in the cache, and potentially evicting lower scoring elements. The algorithm as a whole can be viewed as follows:

\section{PBPS with Hierarchy Algorithm}

1. For each user request $\mathrm{UEC}_{\mathrm{j}}$ with Service Level $\mathrm{L}_{\mathrm{i}}$

2. If $\mathrm{UEC}_{\mathrm{j}}$ is present in one of the $\mathrm{VMs}$ (cache hit)

3. Update hit score of $\mathrm{UEC}_{\mathrm{j}}$ based on $\mathrm{L}_{\mathrm{i}}$ and calculate new overall score using equation (2)

4. Update content of $\mathrm{UEC}_{\mathrm{j}}$ in the cache section for $\mathrm{L}_{\mathrm{i}}$

5. Return;

6. Else if $\mathrm{UEC}_{\mathrm{j}}$ is not present in one of the $\mathrm{VMs}$ (cache miss)

7. Update miss score of $\mathrm{UEC}_{\mathrm{j}}$ and calculate new overall score using equation (2)

8. Write $\mathrm{UEC}_{\mathrm{j}}$ to the cloud

9. Load balance: select $\mathrm{VM}_{\mathrm{i}}$ with the shortest queue

10. If the $V M_{i}$ section for $L_{i}$ has space for the current $U E C_{j}$ 
11. Insert $\mathrm{UEC}_{\mathrm{j}}$ into the $\mathrm{VM}_{\mathrm{i}}$ 's cache section for $\mathrm{L}_{\mathrm{i}}$

12. Return;

13. Else if the $\mathrm{VM}_{\mathrm{i}}$ is full

14. Find $\mathrm{UEC}_{\mathrm{k}}$ which has the lowest score from the cache section for $\mathrm{L}_{\mathrm{i}}$

15. Evict $\mathrm{UEC}_{\mathrm{k}}$ from the $\mathrm{VM}_{\mathrm{i}}$ 's cache section for $\mathrm{L}_{\mathrm{i}}$

16. Write $\mathrm{UEC}_{\mathrm{k}}$ to the cloud

17. Insert $\mathrm{UEC}_{\mathrm{j}}$ into the $\mathrm{VM}_{\mathrm{i}}$ 's cache section for $\mathrm{L}_{\mathrm{i}}$

18. Return;

19. Update all other UECs in the cache using equation (2) with total number request parameter updated

\subsection{Reverse Random Marking (RRM)}

Reverse Random Marking involves all new additions to the cache being entered as unmarked. Once an element in the cache is accessed, meaning there is a cache hit, that element is marked. Any time an eviction is needed, an element is randomly chosen from the unmarked elements in the cache and is removed to make space for a cache insertion. This algorithm is included in this paper, as it will be used to judge how well it can be improved with enhancements. Here is an example adaptation for 5G C-RAN.

\section{Reverse Random Marking (RRM) Algorithm}

1. For each user request $\mathrm{UEC}_{\mathrm{j}}$ with $\mathrm{L}_{\mathrm{i}}$

2. If UEC present in one of VMs (cache hit)

3. Update content of $\mathrm{UEC}_{\mathrm{j}}$ 
4. If UEC is unmarked

5. Mark $\mathrm{UEC}_{\mathrm{j}}$

6. Else if $\mathrm{UEC}_{\mathrm{j}}$ is already marked

6. Return

7. Else if $\mathrm{UEC}_{\mathrm{j}}$ not present in one of VMs (cache miss)

8. Write $\mathrm{UEC}_{\mathrm{j}}$ to cloud storage

9. Select $\mathrm{VM}_{\mathrm{i}}$ using shortest queue load balancing algorithm

10. If $\mathrm{VM}_{\mathrm{i}}$ has space for current $\mathrm{UEC}_{\mathrm{j}}$

11. Insert $\mathrm{UEC}_{\mathrm{j}}$ into $\mathrm{VM}_{\mathrm{i}}$ 's cache

12. Else if VM is full

13. If there are unmarked elements

14. Evict random $\mathrm{UEC}_{\mathrm{k}}$ from unmarked elements

15. Else if all elements are marked

16. $\quad$ Evict random $\mathrm{UEC}_{\mathrm{k}}$

17. Write $\mathrm{UEC}_{\mathrm{k}}$ to cloud storage

18. Insert $\mathrm{UEC}_{\mathrm{j}}$ into $\mathrm{VM}_{\mathrm{i}}$ cache

19. Return

4.4 Reverse Random Marking (RRM) with PBPS

The authors of the Reverse Random Marking algorithm performed their work and research around enhancing a prior Random Marking (RM) caching algorithm [17]. 
Though RRM did offer improvements in performance compared to RM, Reverse Random Marking seems to present a few challenges as well. The authors mentioned continually expanding the size of the cache in order to run the algorithm whenever there are no unmarked elements present. However, as cache is a limited resource, it cannot be expanded indefinitely. Furthermore, problems arise when, in the worst case, all elements in the cache are marked, and eviction becomes a question. In light of these challenges, RRM can actually be further enhanced with probability-based popularity scoring.

With PBPS, each UEC is given a score based on the UEC's service level and the number of hits and misses it has received. In RRM, PBPS can be used in managing the cache elements. In terms of marking the elements, a threshold can be set to determine whether an element can be marked or not. Unlike the regular RRM, the newer version of the algorithm will not mark elements simply from being accessed once. RRM with PBPS will only mark elements if they have a score above some set threshold. This threshold is an arbitrary number and can be set high or low depending on the system needs. Furthermore, in the event that all elements in the cache become marked, the threshold can be marginally increased to unmark a number of elements. This way, there will always be unmarked elements present in the cache, from which an element can be chosen at random to evict when needed. Given service level $\mathrm{L}_{\mathrm{i}}$ and marking threshold $\mathrm{M}_{\mathrm{t}}$, the general algorithm for RRM with PBPS can be seen as follows:

\section{RRM with PBPS Algorithm}

1. For each user request $\mathrm{UEC}_{\mathrm{j}}$ with $\mathrm{L}_{\mathrm{i}}$

2. If $\mathrm{UEC}_{\mathrm{j}}$ is present in one of the $\mathrm{VMs}$ (cache hit) 
3. Update content of $\mathrm{UEC}_{\mathrm{j}}$

4. Update hit score of $\mathrm{UEC}_{\mathrm{j}}$ based on service level and calculate new overall score using equation (2)

5. Return;

6. If $\mathrm{UEC}_{\mathrm{j}}$ 's score exceeds $\mathrm{M}_{\mathrm{t}}$, mark $\mathrm{UEC}_{\mathrm{j}}$

7. Else if $\mathrm{UEC}_{\mathrm{j}}$ 's score does not meet threshold, leave $\mathrm{UEC}_{\mathrm{j}}$ unmarked

8. If all UECs are marked, increase $\mathrm{M}_{\mathrm{t}}$ and reevaluate marked and unmarked elements

9. Else if $\mathrm{UEC}_{\mathrm{j}}$ is not present in one of the VMs (cache miss)

10. Update miss score of $\mathrm{UEC}_{\mathrm{j}}$ and calculate new overall score using equation (2)

11. Write $\mathrm{UEC}_{\mathrm{j}}$ to the cloud

12. Load balance: select $\mathrm{VM}_{\mathrm{i}}$ with the shortest queue

13. If $\mathrm{VM}_{\mathrm{i}}$ has space for the current $\mathrm{UEC}_{\mathrm{j}}$

14. Insert $\mathrm{UEC}_{\mathrm{j}}$ into $\mathrm{VM}_{\mathrm{i}}$ 's cache

15. Return;

16. Else if $\mathrm{VM}_{\mathrm{i}}$ is full

17. Pick random $\mathrm{UEC}_{\mathrm{k}}$ from unmarked elements in the cache

18. Evict $\mathrm{UEC}_{\mathrm{k}}$ from the VM's cache

19. Write $\mathrm{UEC}_{\mathrm{k}}$ to the cloud

20. Insert $\mathrm{UEC}_{\mathrm{j}}$ into the VM's cache

21. Return;

22. Update all other UECs in the cache using equation (2) with total number request parameter updated 
This algorithm, combining Reverse Random Marking with a scoring system, has brought forth an interesting revelation in regards to caching for 5G C-RAN. Note that RRM alone was certainly an algorithm not intended to be used for the cellular network setting, at least not well. However, its combination with the probability-based scoring system has now made it a reasonable algorithm that could be used to manage the cache in this particular architecture. This shows that it is possible to take a caching algorithm not originally intended for 5G C-RAN, combine it with a scoring system, and make it a feasible option for cache management.

\subsection{EXD-AHP}

A fourth scheme useful in managing the cache that also uses a type of scoring system is Exponential Delay - Analytical Hierarchical Process, or EXD-AHP for short, which is a combination of preexisting caching schemes $[6,12,13]$. This approach combines both an exponential decay model to determine the recency and frequency of UECs in the cache, as well as an Analytical Hierarchical Process (AHP) matrix to differentiate between service levels. This way, more recent and frequent items are maintained in the cache while less frequent and less recent items are more likely to be evicted, and higher quality of service is given preferential treatment and priority in the cache over the lower quality of service. The EXD-AHP scheme will be simulated along all other caching schemes, and will also newly be tested for latency to better understand 
its performance. In this scheme, the following equation is used to calculate the score of an incoming UEC:

$$
\mathrm{S}_{\mathrm{i}}\left(\mathrm{u}_{\mathrm{i} 1}+\Delta \mathrm{u}\right)=\mathrm{S}_{\mathrm{i}}\left(\mathrm{u}_{\mathrm{i} 1}\right) * \mathrm{e}^{-\mathrm{a} \Delta \mathrm{u}}+\mathrm{W}_{\mathrm{AHP}}
$$

Relatedly, the following equation is used to evaluate the scores of all other UECs in the cache:

$$
\mathrm{S}_{\mathrm{i}}\left(\mathrm{u}_{\mathrm{i} 1}+\Delta \mathrm{u}\right)=\mathrm{S}_{\mathrm{i}}\left(\mathrm{u}_{\mathrm{i} 1}\right) * \mathrm{e}^{-\mathrm{a} \Delta \mathrm{u}}
$$

In the above equations, the scores of UECs are calculated based on the time taken between requests of the same UEC. If a particular UEC is not accessed or requested between the time $u_{i 1}$ up to $u_{i 1}+\Delta u$, the score of that particular UEC in the cache is updated using equation 4. If a particular UEC is being accessed within the allotted time frame, then the score is updated using equation 3. Both equations take into consideraton the exponential decay model, in which the value of the variable "a" can be set higher to focus the model on recency. In the first equation, the analytical hierarchical process matrix is used in part to determine the score of a UEC that is being accessed in a timely manner. AHP will be used in that each service level will be compared with every other service level, and preferential treatment can be given by specifying how many times more important one level is than the other. The typical AHP matrix is then used to calculate a weight which is added as part of the UEC score. Consider Table 1 on the following page, which illustrates the Analytical Hierarchical Process Matrix. The service levels are each given a row and column in the matrix, and are compared to each other in terms of how many times more improtant one level is than the other. In this case, a higher service level is five times more important than the immediately lower service level. The final weights 
are determined after a series of steps. The results inside the matrix are first squared. Each row is then summed to a total vector. The vector containing the totals is then normalized by being divided by the sum of all entries inside the matrix. The aforementioned steps are then repeated until there is no change in the final results vector. That is, the squaring of the matrix results, the summing of the rows into the result vector, and the normalization of the results vector, is repeated until the results vector is unchanged from a previously iterated calculation. Once this has completed, the final weights are used to differentiate each of the service levels. In the cache management scheme, the weight is added as part of the equation used to calculate the score of a UEC that is currently being accessed.

Table 1. Example Analytical Hierarchical Process Matrix with Weights

\begin{tabular}{|c|c|c|c|c|c|}
\hline & SLA 1 & SLA 2 & SLA 3 & SLA 4 & $\mathrm{W}_{\text {AHP }}$ \\
\hline SLA 1 & 1 & $5 / 1$ & $5 / 1$ & $5 / 1$ & 0.579 \\
\hline SLA 2 & $1 / 5$ & 1 & $5 / 1$ & $5 / 1$ & 0.281 \\
\hline SLA 3 & $1 / 5$ & $1 / 5$ & 1 & $5 / 1$ & 0.102 \\
\hline SLA 4 & $1 / 5$ & $1 / 5$ & $1 / 5$ & 1 & 0.043 \\
\hline
\end{tabular}

The following is the overview of the entire algorithm:

\section{EXD-AHP Algorithm}

1. For each user request $\mathrm{UEC}_{\mathrm{j}}$ with $\mathrm{L}_{\mathrm{i}}$

2. Calculate new score with Eq. (3) for $\mathrm{UEC}_{\mathrm{j}}$ since it will be potentially accessed

3. Update score of all other UECs in the cache using Eq. (4) as they are not accessed 
4. If $\mathrm{UEC}_{\mathrm{j}}$ is present in one of VMs (cache hit)

5. Update score and content of $\mathrm{UEC}_{\mathrm{j}}$

6. Return

7. Else if $\mathrm{UEC}_{\mathrm{j}}$ is not present in one of $\mathrm{VMs}$ (cache miss)

8. Write $\mathrm{UEC}_{\mathrm{j}}$ to cloud storage

9. Select $\mathrm{VM}_{\mathrm{i}}$ using shortest queue load balancing algorithm

10. If $\mathrm{VM}_{\mathrm{i}}$ has space for the current $\mathrm{UEC}_{\mathrm{j}}$

11. Insert $\mathrm{UEC}_{\mathrm{j}}$ into $\mathrm{VM}_{\mathrm{i}}$ 's cache

12. Return

13. Else if $\mathrm{VM}_{\mathrm{i}}$ is full

14. If $\mathrm{UEC}_{\mathrm{j}}$ score is more than smallest score present in cache

15. Evict lowest scoring entries whose sum is less than $\mathrm{UEC}_{\mathrm{j}}$ score

16. Write evicted UECs to the cloud

17. Else if $\mathrm{UEC}_{\mathrm{j}}$ score is smaller than lowest score

18. Evict $\mathrm{UEC}_{\mathrm{k}}$ with smallest score

19. Write $\mathrm{UEC}_{\mathrm{k}}$ to the cloud

20. Insert $\mathrm{UEC}_{\mathrm{j}}$ into $\mathrm{VM}_{\mathrm{i}}$ cache

21. Return 


\subsection{Baseline Comparisons}

In order to assess the benefit and performance of the various caching schemes, it is useful to compare them to a known classic scheme. Comparison will therefore be made with Least Frequently Used (LFU). LFU involves keeping track of the number of times a particular UEC element in the cache is accessed. In the event that eviction needs to be performed when the cache is full and space is needed for new elements, the element with the lowest number of accesses will be evicted [17].

An important aspect of LFU should be taken into consideration in the context of cellular network caching. Least Frequently Used simply counts the number of accesses an element gets in the cache. It does not have a mechanism to differentiate between requests of different service levels. Regardless of the quality of service, a UEC will be given an increment towards the number of accesses when the request for that particular UEC comes through. Because of this, the performance of LFU is related to the traffic distribution itself and not related to or based directly on service levels. Least Frequently Used will treat requests across all the service levels the same. With that said, LFU can still be used as a caching mechanism in 5G C-RAN. In the worse case scenario, the traffic load is equally distributed between all service levels. That is, there are roughly an equal number of users in each service level. In this case, performance from LFU would be poor in that not much differentiation would be seen between service levels. In the best case scenario, the traffic load is unevenly distributed between service levels, with better service levels having more user requests. In this case, since LFU is related to the traffic distribution, a clear distinction would be seen in performance based on service levels. For the purposes of this 
project, the best case scenario for LFU is used, which showcases the best scenario for LFU in terms of performance, as a base comparison. This knowledge about LFU also indicates the importance of needing more tailored algorithms to use in 5G C-RAN, which can offer both a proper way of managing the cache in any type of traffic distribution or workload, and consistently making appropriate differentiations between quality of services. 


\section{PERFORMANCE EVALUATION}

In order to assess and analyze the performance of these algorithms, they were implemented, tested, and compared through a simulator called CloudSim. CloudSim is a programmable simulator based in Java that allows users to simulate all manner of cloud and data center elements as well as set up their own policies for resource management [2]. The algorithms that were tested, and the settings in the simulation, are summarized in the following tables.

Table 2. Simulated Algorithms

\begin{tabular}{|l|l|l|}
\hline \multicolumn{1}{|c|}{ Acronym } & \multicolumn{1}{|c|}{ Algorithm } & \multicolumn{1}{c|}{ Details } \\
\hline LFU [17] & Least Frequently Used & Uses Counter for \# Accesses for \\
& & UEC \\
\hline EXD-AHP [12, & $\begin{array}{l}\text { Exponential Decay with } \\
\text { Analytical Hierarchical } \\
\text { Process }\end{array}$ & Score UEC Elements Using \\
& Equations (3) and (4) \\
\hline RRM [17] & Reverse Random Marking & Marking and Unmarking UEC \\
\hline RRM + PBPS & Reverse Random Marking \\
(new) & Eith Probability-Based & RRM Made Fit for 5G with \\
& Popularity Scoring & PBPS Equation (2) \\
\hline
\end{tabular}




\begin{tabular}{|l|l|l|}
\hline PBPS (new) & $\begin{array}{l}\text { Probability-Based Popularity } \\
\text { Scoring }\end{array}$ & $\begin{array}{l}\text { Scores UEC based on Equation } \\
(2)\end{array}$ \\
\hline PBPS + Hierarchy & $\begin{array}{l}\text { Probability-Based Popularity } \\
\text { Scoring with Hierarchy }\end{array}$ & $\begin{array}{l}\text { Scoring UEC based on Equation } \\
\text { (new) }\end{array}$ \\
\end{tabular}

Table 3. Simulation Parameters and Values

\begin{tabular}{|c|c|}
\hline Parameter & Value \\
\hline Number of Hosts & 1 \\
\hline Number of VMs on Each Host & 4 \\
\hline Virtual Machine Cache Sizes & $1250 \mathrm{MB} ; 2500 \mathrm{MB} ; 3750 \mathrm{MB} ; 5000 \mathrm{MB}$ \\
\hline Network Bandwidth & $1 \mathrm{Gbps}$ \\
\hline Arrival Rate of UECs into Simulation & $1400 \mathrm{UEC} / \mathrm{sec}$ \\
\hline UEC Size & $200 \mathrm{~KB}$ \\
\hline $\begin{array}{l}\text { Number of Distinct Users in Cellular } \\
\text { Network }\end{array}$ & 25,500 \\
\hline Quality of Service Levels & Description of QoS \\
\hline SLA 1 & $\begin{array}{l}\text { Highly Mobile } \\
\text { Premium }\end{array}$ \\
\hline
\end{tabular}




\begin{tabular}{|c|c|}
\hline SLA 2 & $\begin{array}{c}\text { Less Mobile } \\
\text { Premium }\end{array}$ \\
\hline SLA 3 & $\begin{array}{c}\text { Highly Mobile } \\
\text { Basic }\end{array}$ \\
\hline SLA 4 & $\begin{array}{c}\text { Less Mobile } \\
\text { Basic }\end{array}$ \\
\hline & PBPS Hit Rewards \\
\hline SLA 1 & 1 \\
\hline SLA 2 & 0.75 \\
\hline SLA 3 & 0.5 \\
\hline SLA 4 & 0.25 \\
\hline & Cache Partitioning for Hierarchy \\
\hline SLA 1 & $70 \%$ of Cache \\
\hline SLA 2 & $20 \%$ of Cache \\
\hline SLA 3 & $8 \%$ of Cache \\
\hline SLA 4 & $2 \%$ of Cache \\
\hline
\end{tabular}




\begin{tabular}{|c|c|}
\hline & Analytical Hierarchical Process Weights \\
\hline SLA 1 & 0.58 \\
\hline SLA 2 & 0.28 \\
\hline SLA 3 & 0.10 \\
\hline SLA 4 & 0.04 \\
\hline & Traffic Distribution \\
\hline SLA 1 & $52 \%$ \\
\hline SLA 2 & $26 \%$ \\
\hline SLA 3 & $13 \%$ \\
\hline SLA 4 & $9 \%$ \\
\hline Threshold Increase for RRM+PBPS & $10 \%$ \\
\hline EXD Parameter "a" & $10^{-3}$ \\
\hline Simulation Run Time & 300 Seconds (5 Minutes) \\
\hline
\end{tabular}

Various metrics are used to compare and analyze the algorithms, including hit rates, average request latency, average number of cloud writes per request, and network traffic as a result of misses. 


\subsection{Hit Rates}

Hit rates can simply be defined as follows for each service level $\mathrm{L}_{\mathrm{i}}$ :

$$
\text { Cache Hit Rate } \mathrm{L}_{-} \mathrm{i}=\frac{\# \text { of UEC Hits on } L_{-} i}{\# \text { of UEC Requests from } L_{-} i}
$$

Figures 5 through 8 illustrate how hit rates are supported for each of the four service levels in the simulation, and how the hit rates change and are affected by cache size. When the cache size is small, as seen in the first two charts, EXD-AHP tends to provide the best preferential treatment to the best service level, SLA1. However, it also provides close to no hits on the rest of the service levels and shows a starvation in SLA2, SLA3, and SLA4. PBPS alone also shows a similar pattern in hit rates compared to EXD-AHP, though with a slightly lower hit rate on the best service level. The tradeoff in performance is seen with PBPS + Hierarchy. The PBPS + Hierarchy algorithm offers the best distribution of the cache hits, so each level has a reasonable number of hits while still being differentiated based on service quality. However, the hit rate on the best service level, SLA1, is much lower with PBPS + Hierarchy compared to EXD-AHP or PBPS. RRM maintains the lowest hit rate, but has been improved as RRM + PBPS. With the inclusion of ProbabilityBased Popularity Scoring, RRM has been enhanced, with its new combination offering substantially better results than the previous RRM alone, including triple the number of hits on the best service level. Lastly, Least Frequently Used has a good hit rate on the best service level, but like EXD-AHP and PBPS, tends to neglect the other lower three service levels. It should be noted that LFU has another weakness in that it does not appear to 
make very good differentiation between the lower half of service levels, namely SLA3 and SLA4. The hit rates appear to be almost identical for the two lowest service levels.

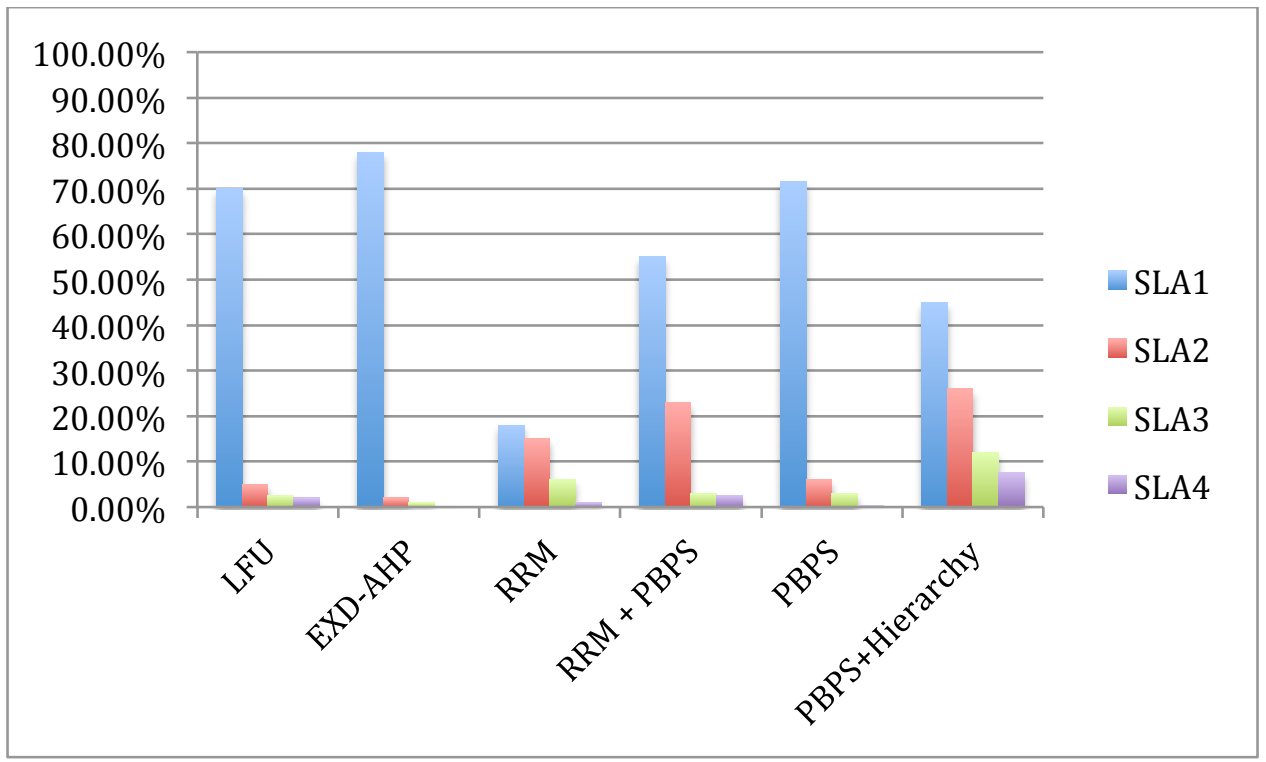

Figure 5. Hit Rates with Cache Size $1250 \mathrm{MB}$

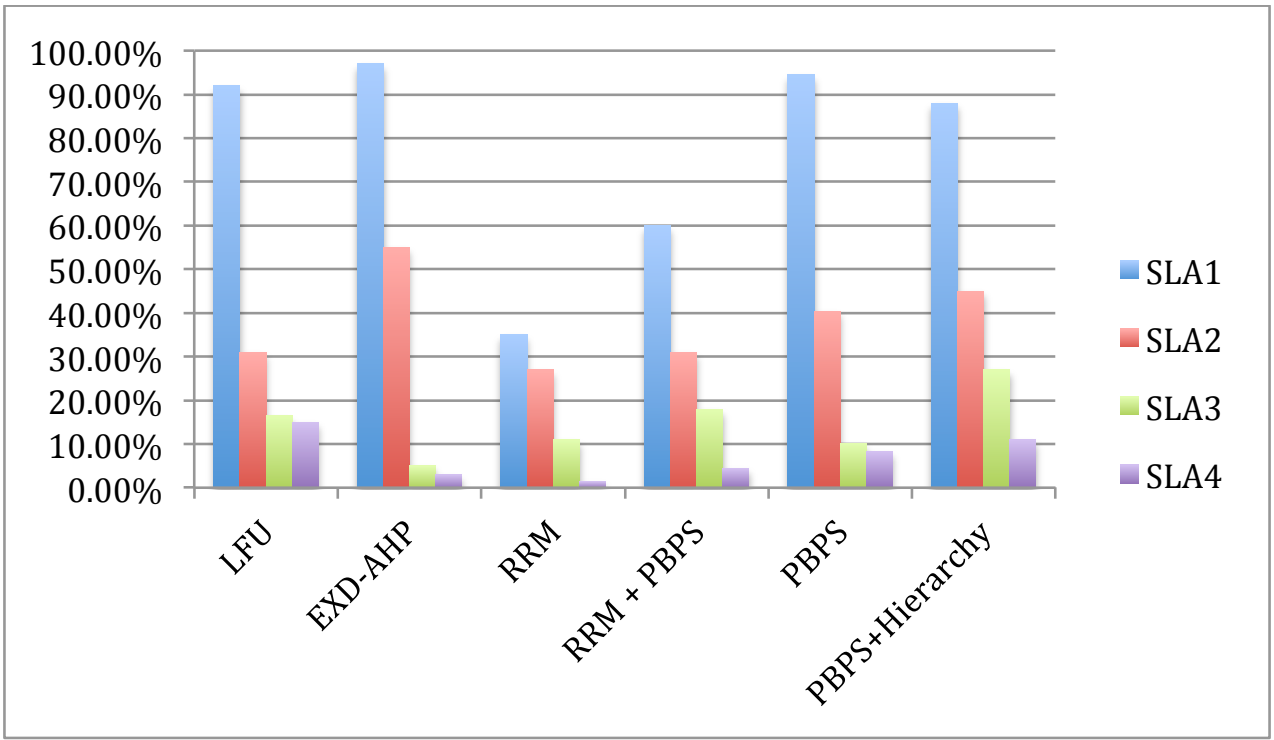

Figure 6. Hit Rates with Cache Size $2500 \mathrm{MB}$ 
With larger cache sizes, more UECs can be accommodated and therefore hit rates, in general, rise. There are a few things to note, however. Larger cache sizes allow for PBPS + Hierarchy to potentially offer a $100 \%$ hit rate on the best service level, as more of the cache can be dedicated to storing SLA1 UECs, if not all. However, as seen in the graphs below, EXD-AHP tends to offer better performance in terms of hit rates on SLA2 and SLA3 in the $3750 \mathrm{MB}$ cache size, which are now surpassing the hierarchical approach. However, the hierarchical approach consistently offers an appreciable distribution of hits.

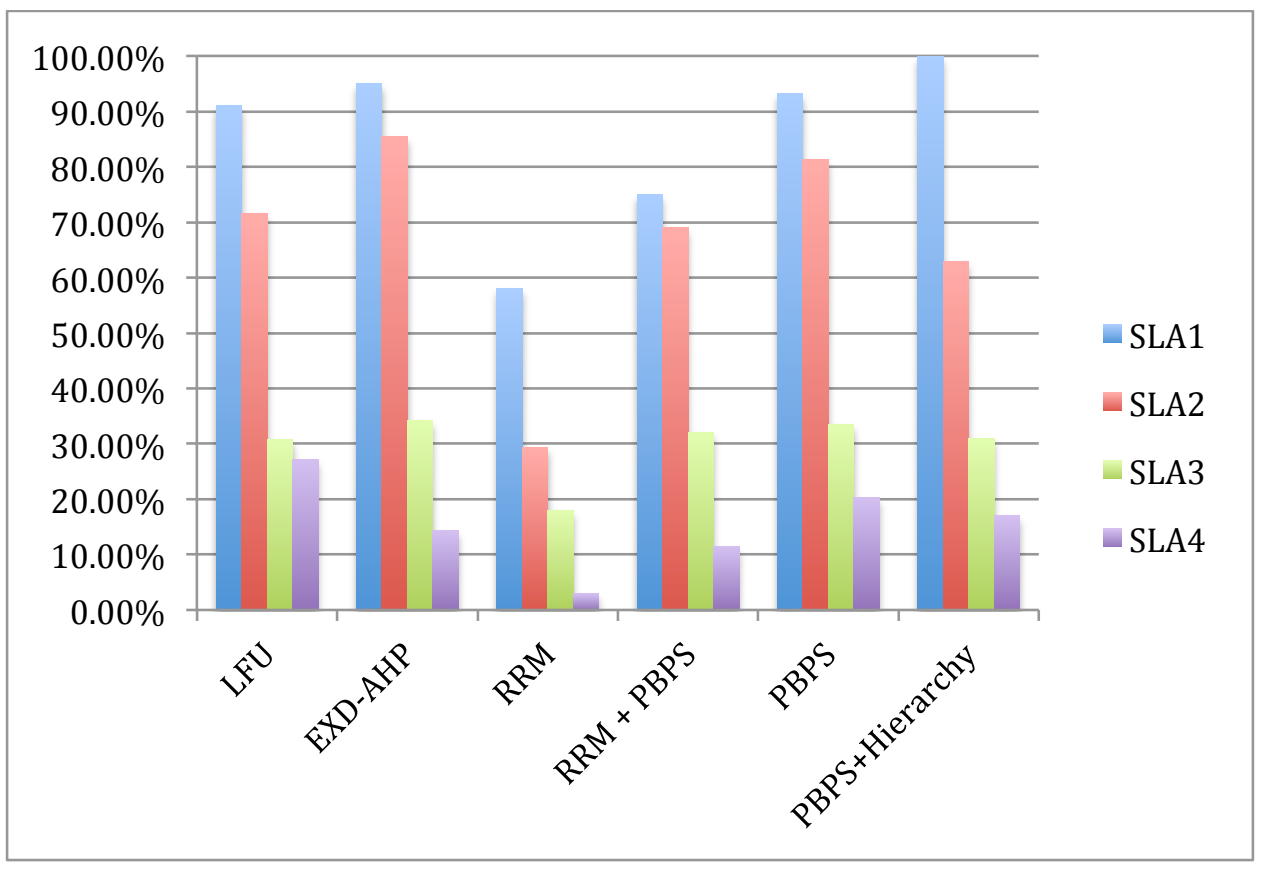

Figure 7. Hit Rates with Cache Size $3750 \mathrm{MB}$ 


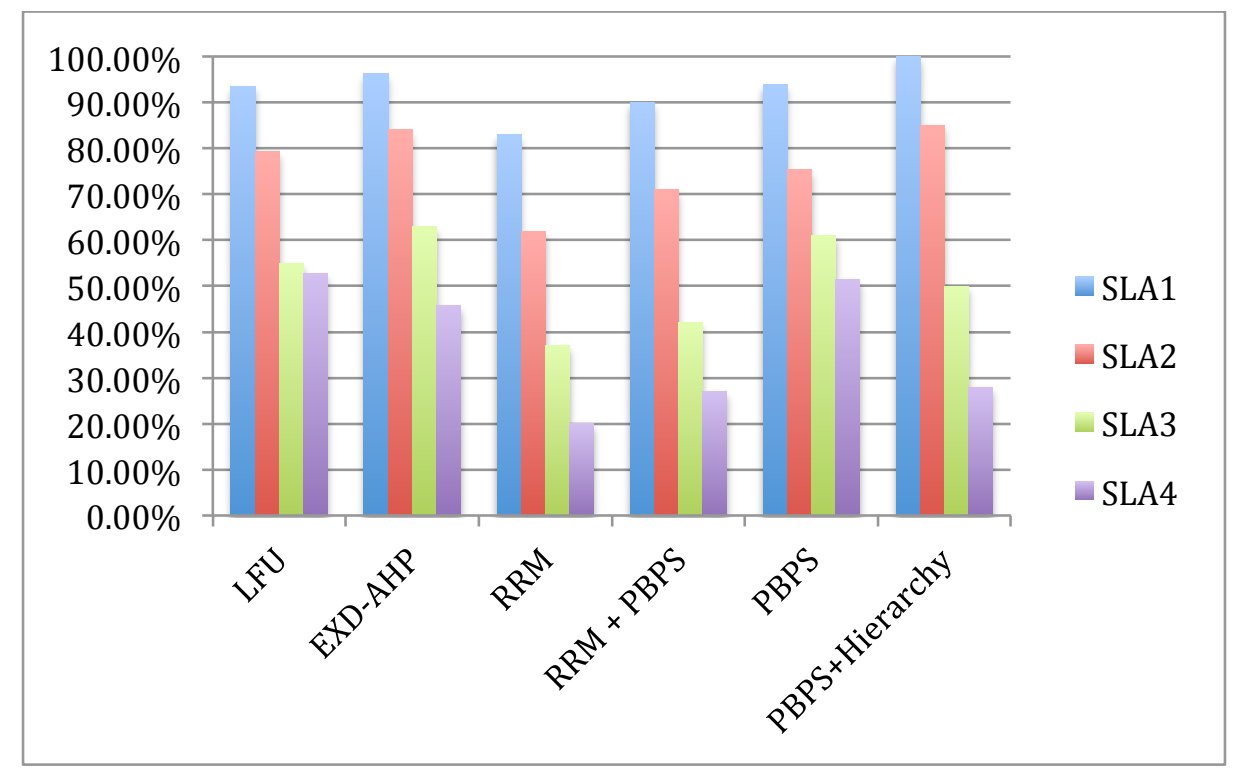

Figure 8. Hit Rates with Cache Size $5000 \mathrm{MB}$

Lastly, it should be noted that PBPS on its own is certainly an acceptable algorithm, but performs better and gives better distribution with the hierarchy in place. RRM all around tends to be the least impressive in terms of performance, but can be greatly improved with incorporating PBPS at any cache size. The enhanced RRM + PBPS then becomes an algorithm that is better suited for this type of architecture. LFU's lack of proper differentiation between the lower service levels again emphasizes the reason why different, more tailored caching schemes are needed for $5^{\text {th }}$ Generation Cloud Radio Access Networks. Even in its best scenario, LFU is not sufficient enough for these newer performance necessities and demands. 


\subsection{Miss Rates}

Miss rates can similarly be defined as follows for each service level $\mathrm{L}_{\mathrm{i}}$ :

$$
\text { Cache Miss Rate } L_{-} \mathrm{i}=\frac{\# \text { of UEC Misses on } L_{-} i}{\# \text { of UEC Requests from } L_{-} i}
$$

The following graphs demonstrate how the miss rates amongst the six algorithms vary, and how the four service levels are supported on different sizes of VM cache. Since the miss rate is proportional to the hit rate, similar patterns are visible. LFU and EXD-AHP have astounding miss rates on lower service levels, and RRM in general has a balance between higher miss rates and distribution that is too close and not well as well differentiated among service levels. PBPS and, even better, PBPS + Hierarchy both show better results in the form of lower miss rates for smaller cache sizes. These two algorithms would be the better options to take given smaller caches. Also note that the hit rate on RRM + PBPS has been significantly improved over the older RRM alone.

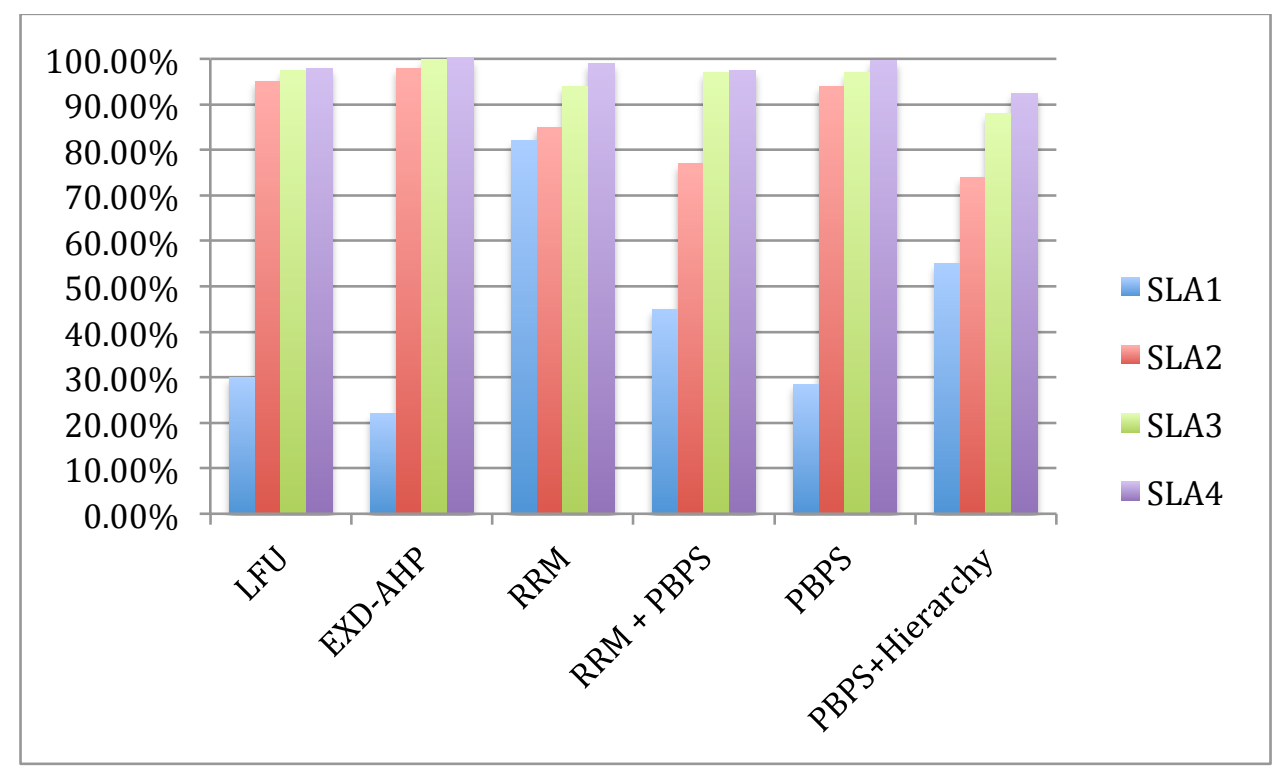

Figure 9. Miss Rate with Cache Size $1250 \mathrm{MB}$ 


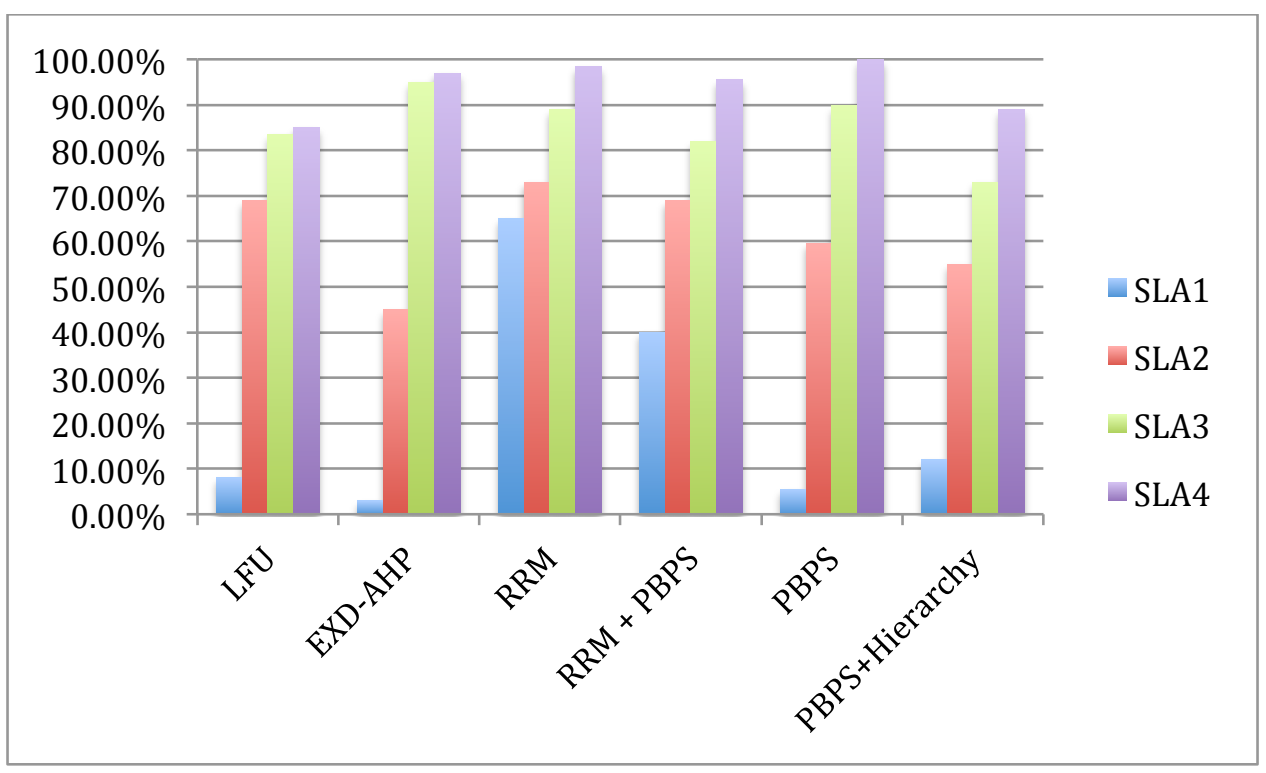

Figure 10. Miss Rate with Cache Size 2500 MB

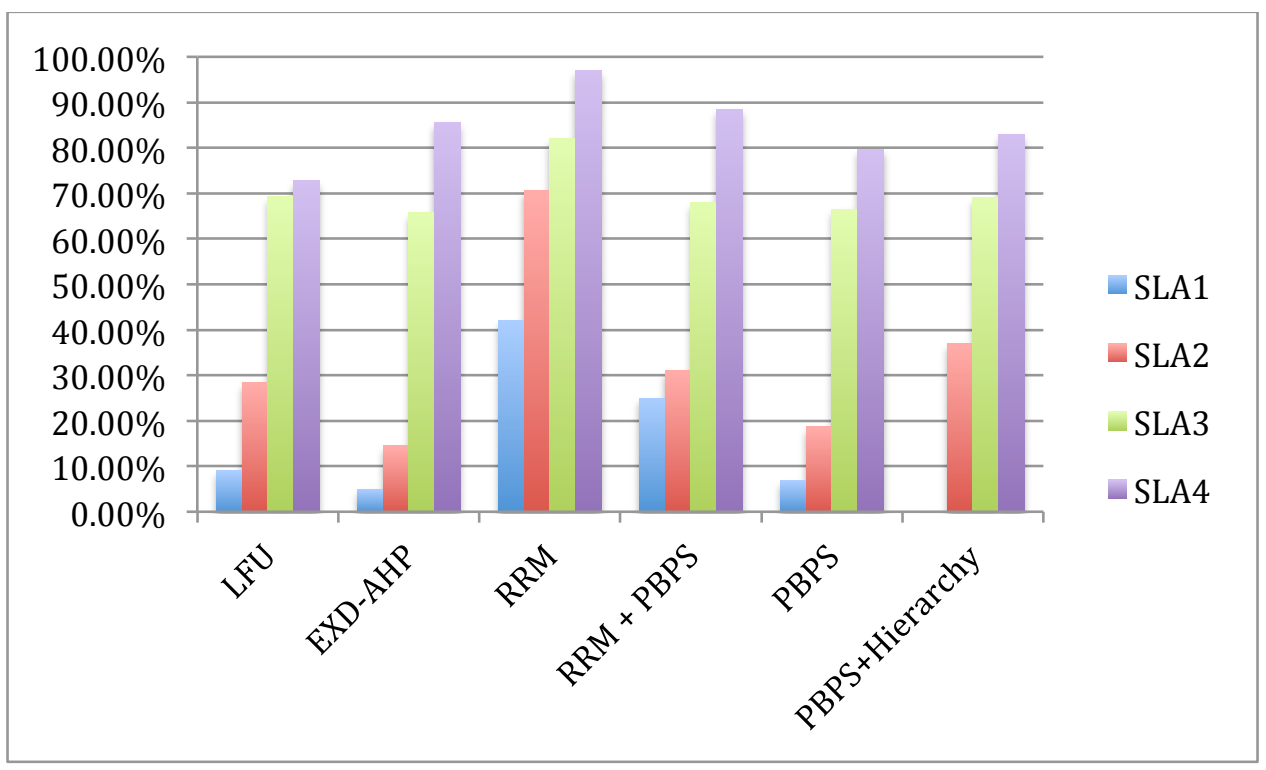

Figure 11. Miss Rate with Cache Size 3750 MB 
These larger cache sizes indicate a preference of different algorithms than the smaller caches. With a larger cache, EXD-AHP offers one of the best overall miss rates, whereas PBPS + Hierarchy brings the miss rate down to zero for the highest service level in the cache sizes of $3750 \mathrm{MB}$ and $5000 \mathrm{MB}$.

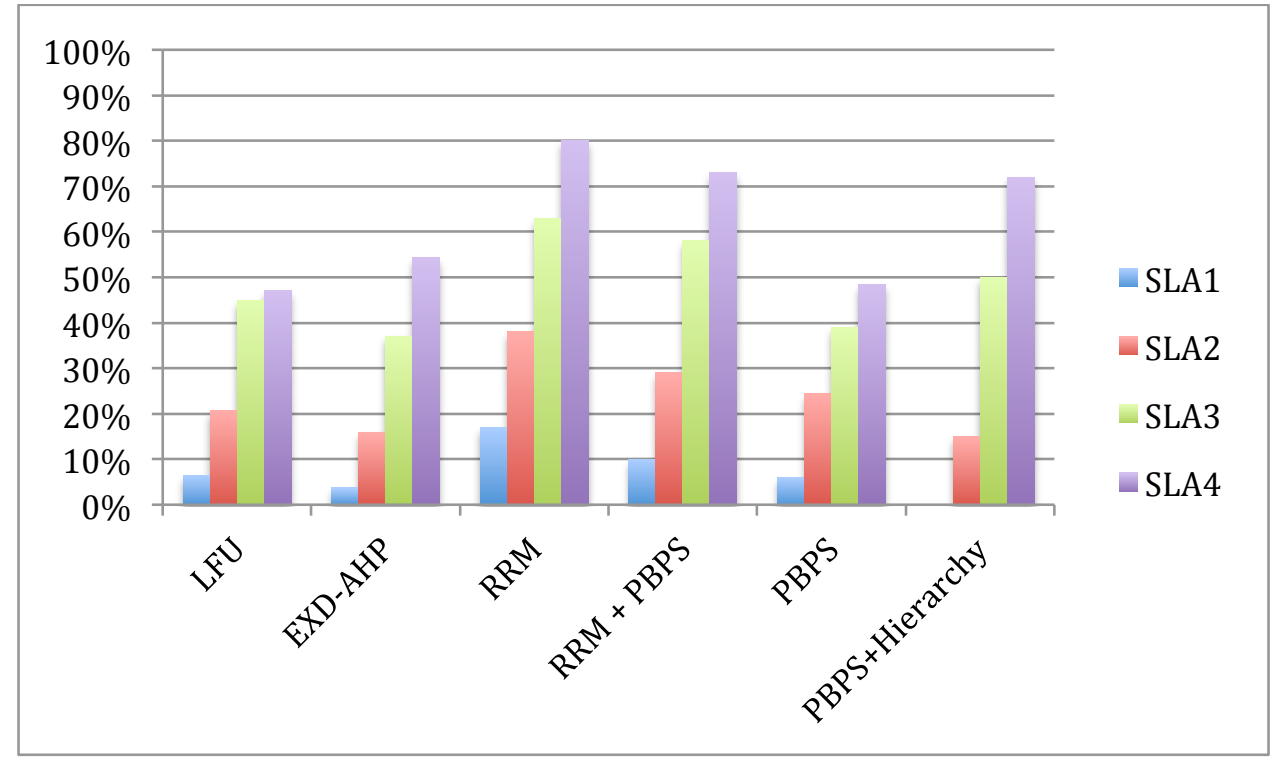

Figure 12. Miss Rate with Cache Size $5000 \mathrm{MB}$

\subsection{Latency}

Another metric used in evaluating the performance of all of the algorithms is latency. Specifically, the latency will be analyzed in terms of how long it takes to process a hit or a miss in the system. In the simulation, latency regarding hits and misses are judged separately, but their average is taken to analyze the general performance. With hits, the latency is simply the amount of time it takes in accessing and updating the UEC in the cache. With misses, the latency is the collective time it takes to 
evict an item from the cache if needed, write the evicted item to the cloud, and write the incoming UEC to the cache and cloud. Misses, therefore, have to take into consideration the transmission time across the data center network. Due to this difference, hits tend to have lower latency and misses tend to take much longer. The following graphs illustrate the average latency per request for each of the algorithms in subsequent cache sizes.

In terms of this particular metric, service is considered better if it is faster. As a result, lower values for latency are preferred in a caching scheme for 5G Cloud-RAN. EXD-AHP offers the lowest latency on the best service level, but PBPS + Hierarchy offers better latency results on the lower service levels while still properly differentiating the different quality of services.

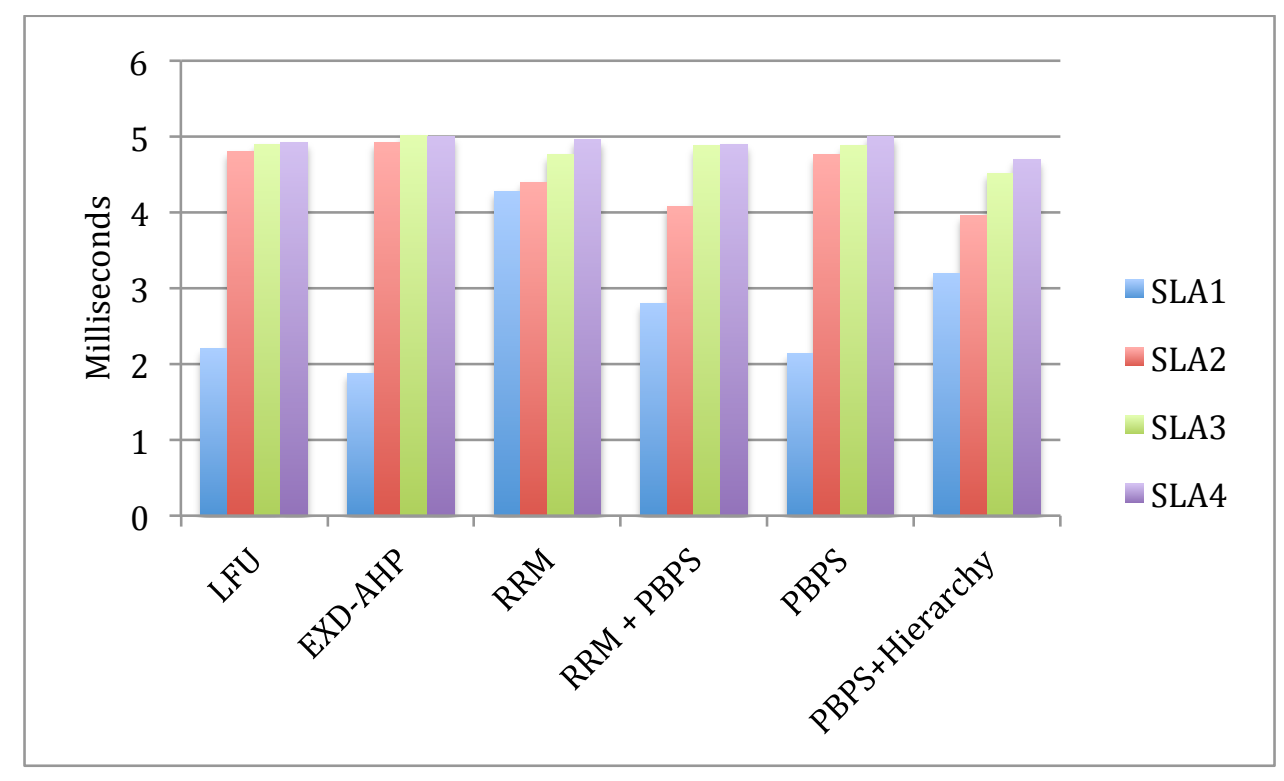

Figure 13. Average Request Latency with Cache Size 1250 MB 


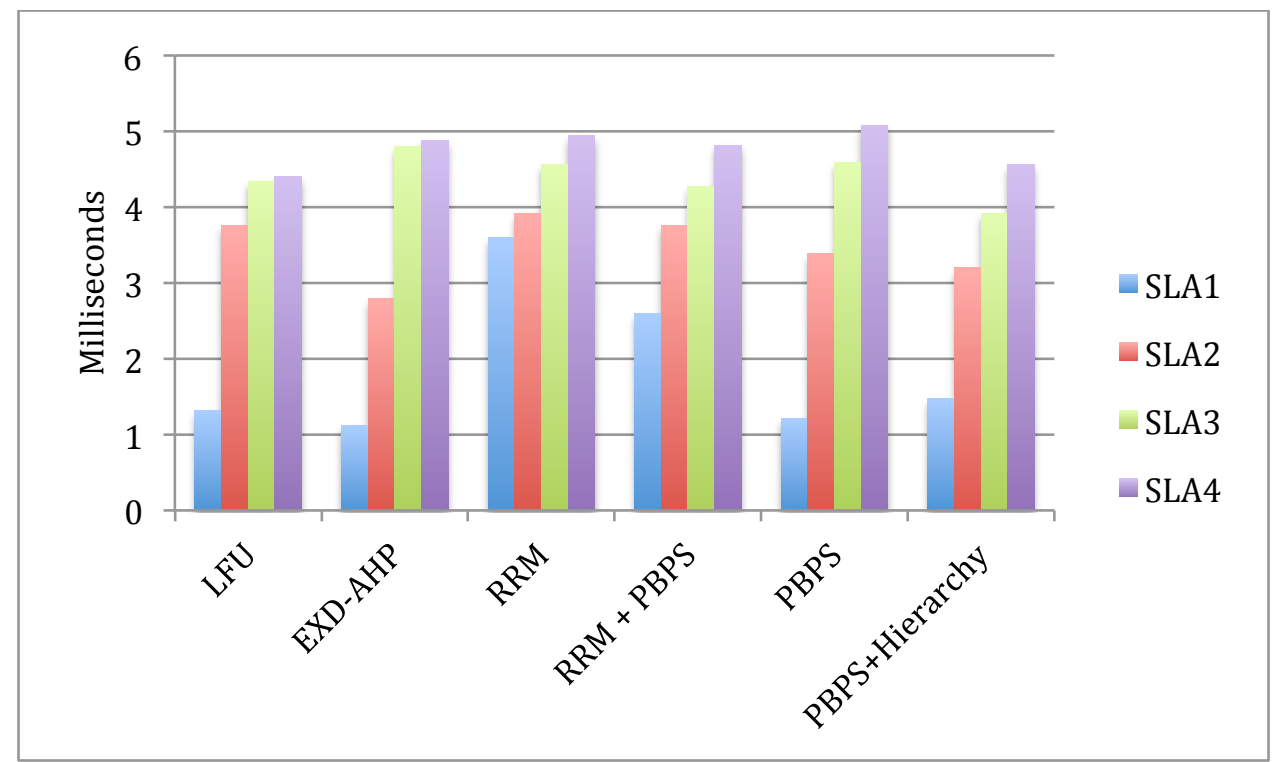

Figure 14. Average Request Latency with Cache Size 2500 MB

As seen in the larger two cache sizes in Figures 15 and 16, PBPS, PBPS + Hierarchy, and EXD-AHP all offer better latency values overall amongst the various service levels than the other algorithms. PBPS + Hierarchy, due to the partitioning of the cache for various service levels, has the potential to offer the minimal amount of latency in the best service level. 


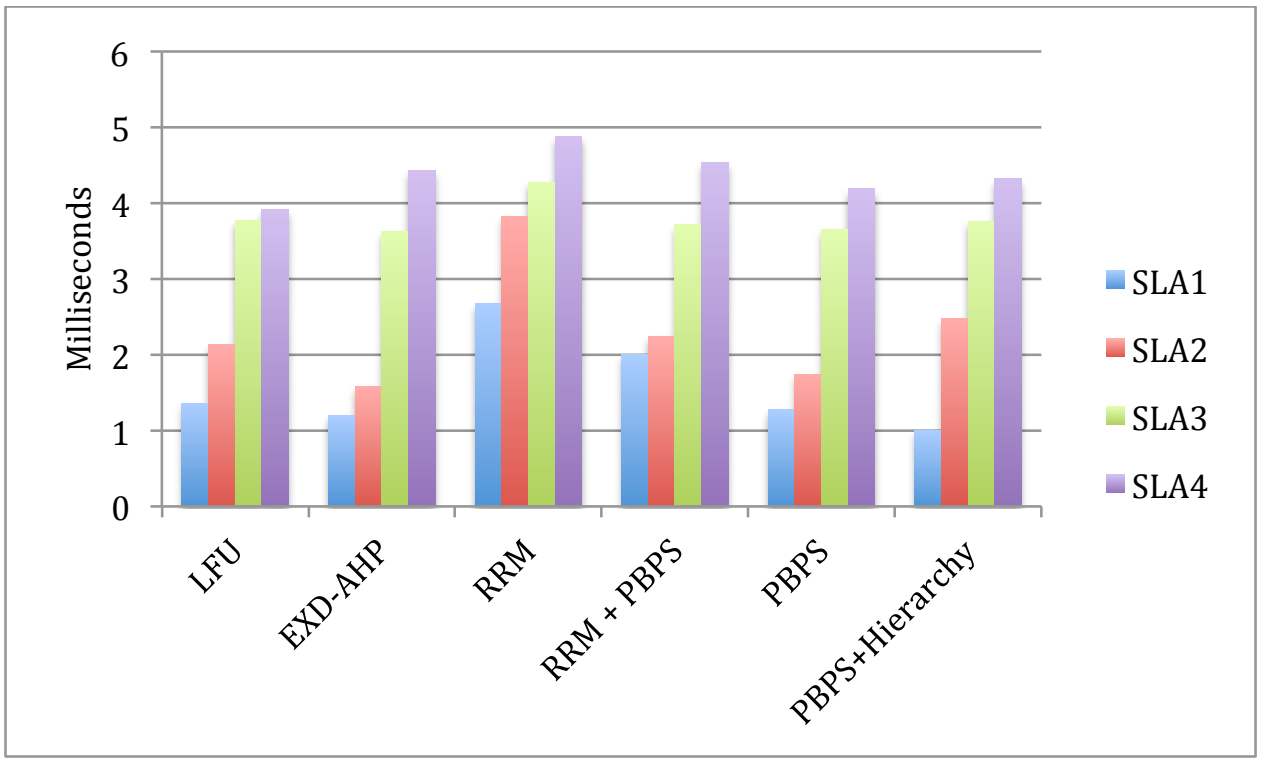

Figure 15. Average Request Latency with Cache Size 3750 MB

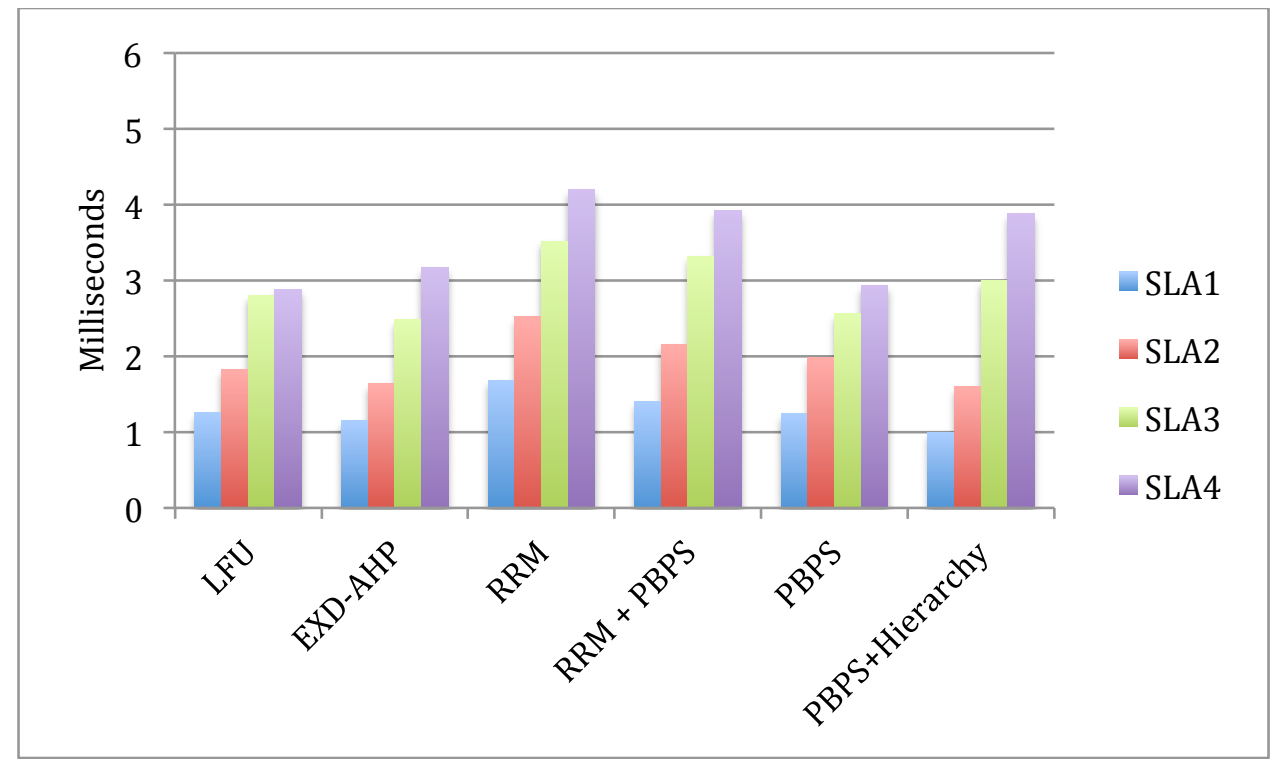

Figure 16. Average Request Latency with Cache Size 5000 MB 
RRM does not offer substantial improvement in the latency of each request even with larger cache sizes, but its combination with PBPS has proven to be quite useful. LFU in larger cache sizes, though seemingly competitive, has trouble again differentiating between the lower two service levels.

\subsection{Cloud Writes}

The number of cloud writes based on the service levels is another metric useful in comparing and understanding the algorithms better. Cloud writes are represented as an average number of writes to cloud storage per UEC request in a particular algorithm. That is, whenever there is a cache hit, there is no write to the cloud and so the number of cloud writes is 0 . When there is a cache miss, the evicted UEC must be written to the cloud and the incoming UEC must also be written to the cloud, resulting in 2 writes to the cloud. The average number of writes per request, therefore, will be a number between 0 and 2. The number of cloud writes can be calculated using the following formula.

$$
\text { Average \# L_i Cloud Writes }=\frac{\# \text { of Cloud Writes in } L_{-} i}{\# \text { of UEC Arrivals in } L_{-} i}
$$

Smaller cache sizes demonstrate that EXD-AHP offers the fewest writes on the best service level. However, it has some of the highest number of writes on all the three other service levels. PBPS is comparable in the same aspects, and like EXD-AHP, it does not differentiate amongst the other lower service levels very well in terms of writes. With writes, PBPS + Hierarchy and even RRM + PBPS appear to offer better numbers in the performance of some of the lower service levels. 


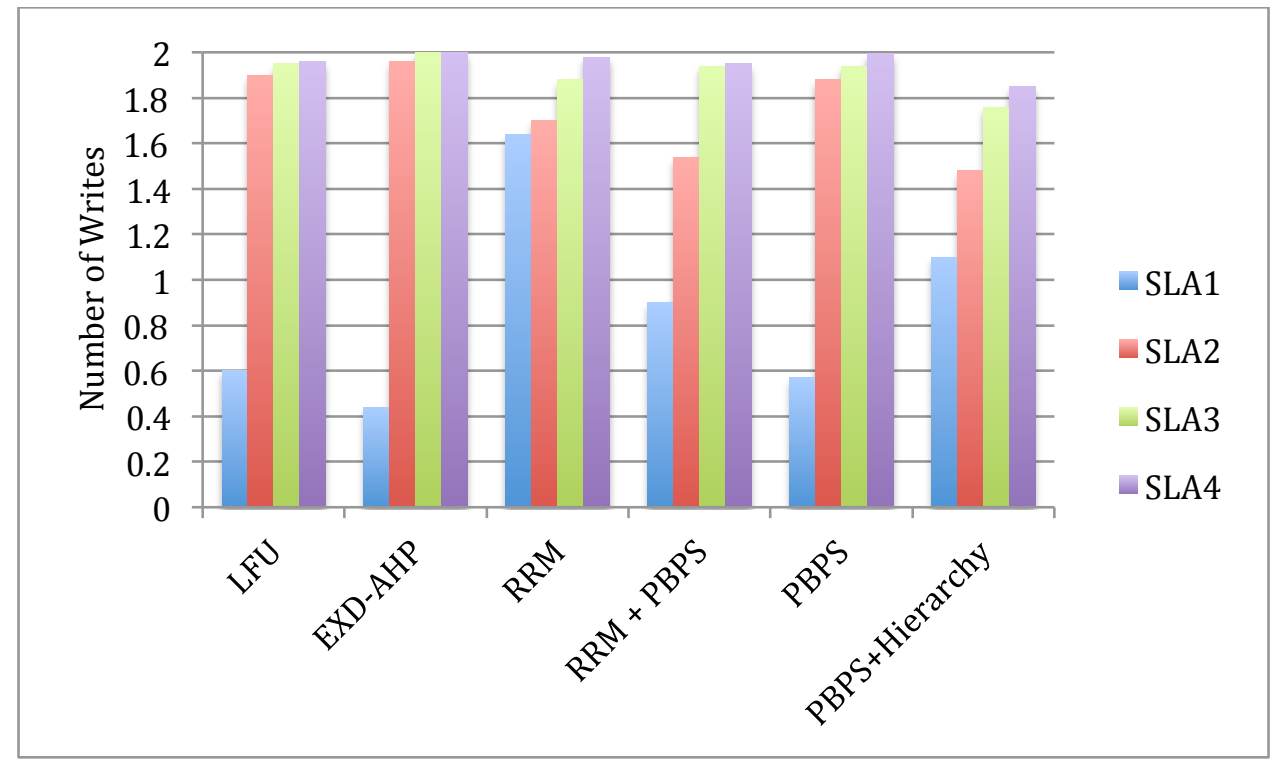

Figure 17. Average Number of Cloud Writes with Cache Size 1250 MB

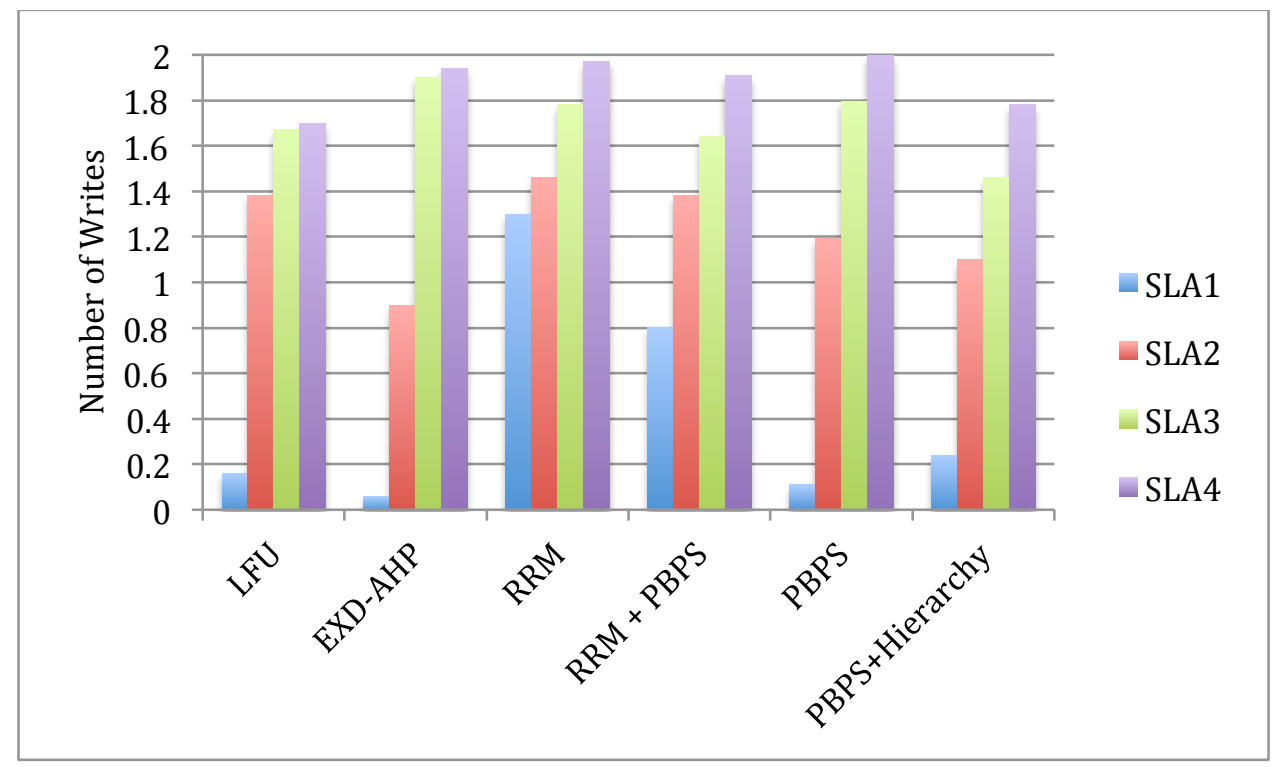

Figure 18. Average Number of Cloud Writes with Cache Size 2500 MB 
With larger cache sizes, EXD-AHP substantially reduces the writes on the higher half of service levels, as does PBPS following in second. However, PBPS + Hierarchy offers potentially no writes on the best service level, since enough of the cache could be allotted to SLA1 to store all SLA1 users.

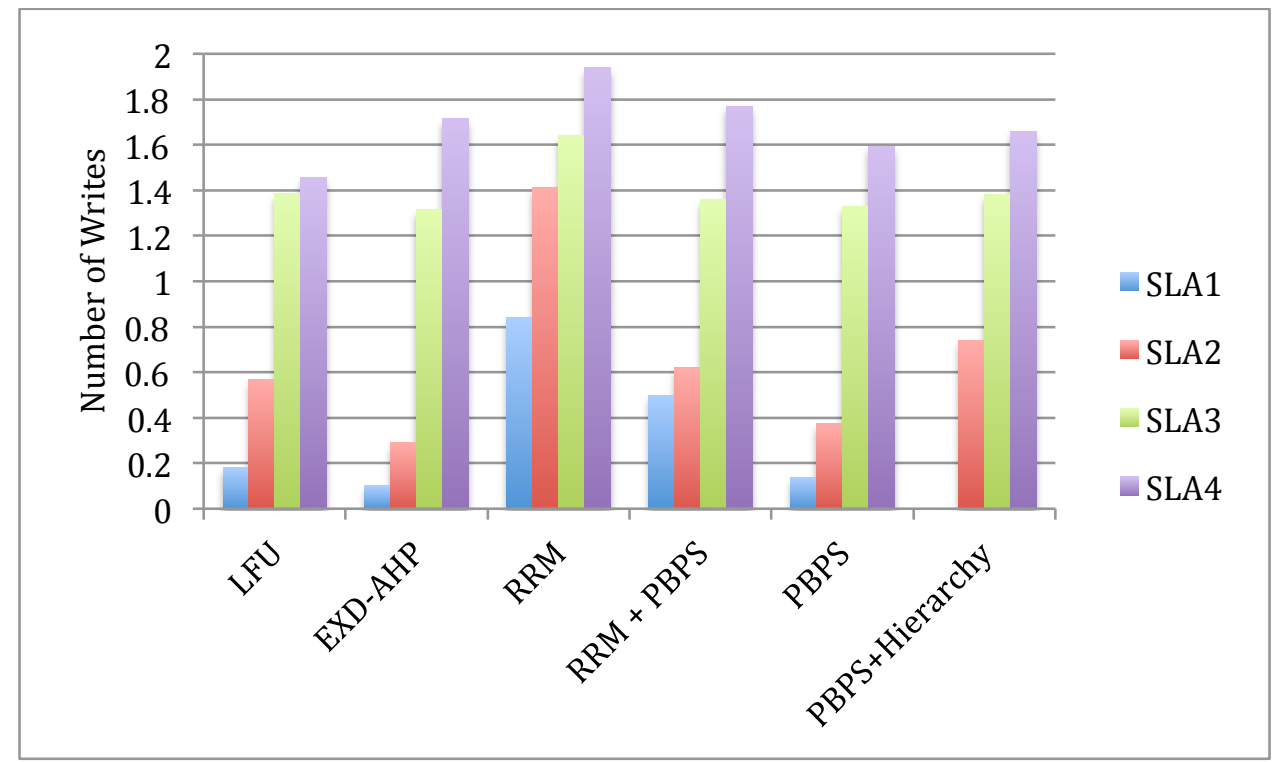

Figure 19. Average Number of Cloud Writes with Cache Size 3750 MB 


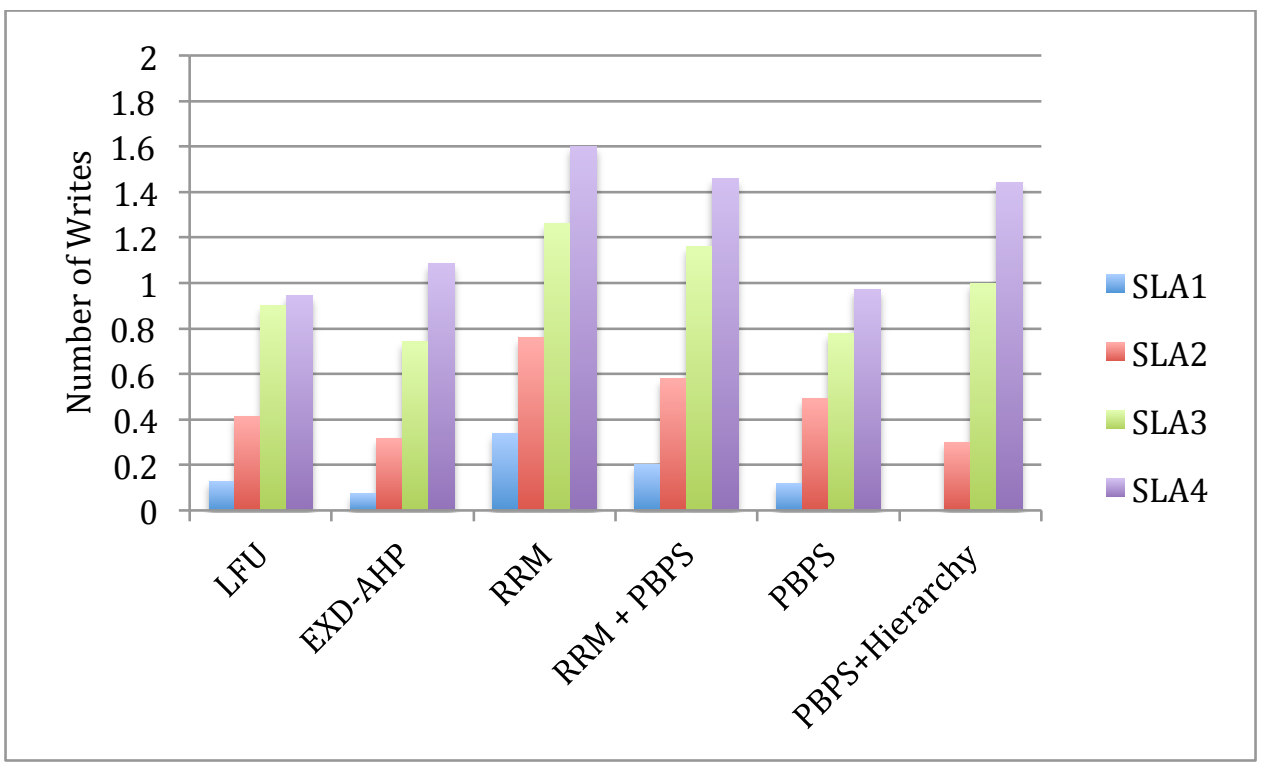

Figure 20. Average Number of Cloud Writes with Cache Size 5000 MB

RRM may be somewhat acceptable at the smallest cache size, but in general its combination with PBPS is preferred to decrease the number of writes. RRM and LFU both show that classic or older caching mechanisms are not good enough for 5G C-RAN, and that better alternatives can be created and are preferred.

\subsection{Network Traffic}

A third metric used to compare the performance of these algorithms is that of network traffic. Whenever there is a cache miss on a UEC request, there is a need to travel across the data center network from the cache to the cloud storage in order to perform the necessary writes. As a result, there is a certain amount of traffic that is 
generated on the network as a result of misses. Therefore, the traffic can be calculated as follows:

$$
\text { Network Traffic }=\left(\# \text { Writes } * 200 \frac{K B}{U E C} * 8 \frac{\text { bits }}{\text { byte }} * 1000 \frac{\text { bytes }}{K B}\right)
$$

After calculating the network traffic, here is the comparative performance of all the algorithms in different cache sizes. With smaller cache sizes, EXD-AHP and PBPS both result in less traffic on the network, resulting in better and faster service, since they offer the best service to the highest service level, SLA, which contains the most number of users. They are followed closely by LFU, PBPS + Hierarchy, and RRM + PBPS.

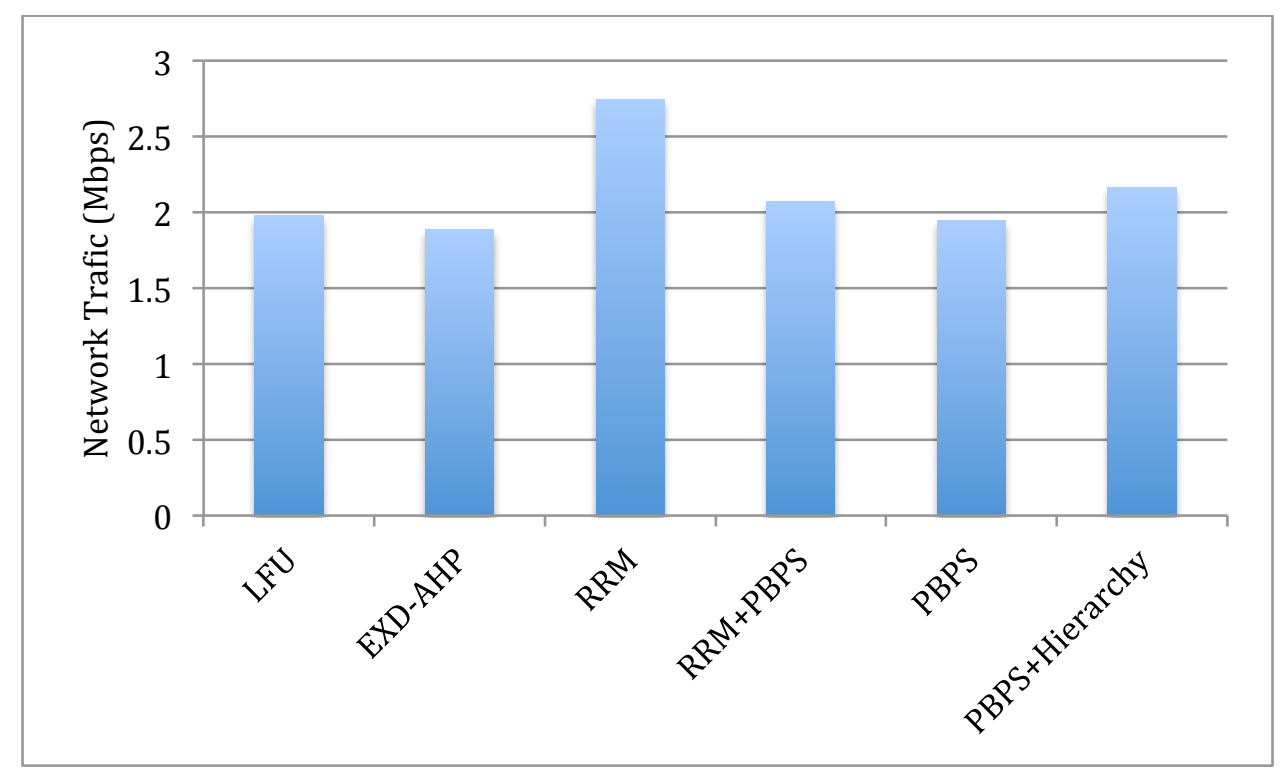

Figure 21. Network Traffic with Cache Size 1250 MB 


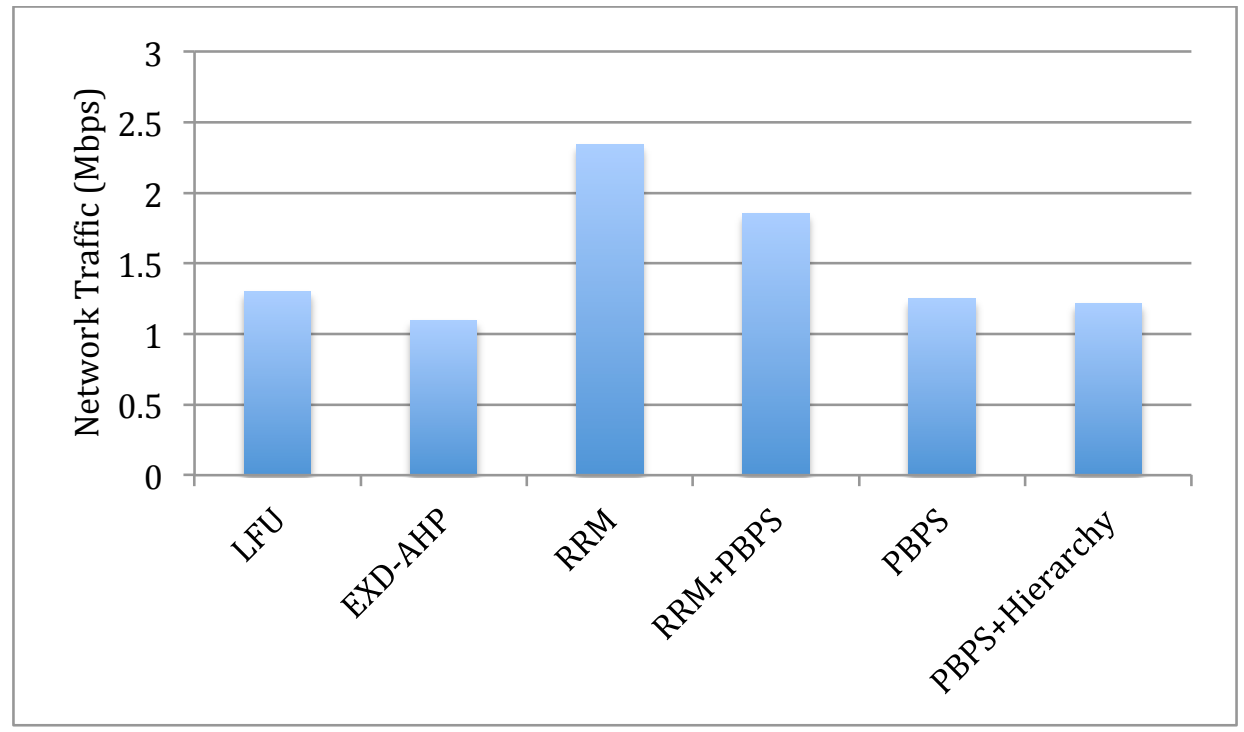

Figure 22. Network Traffic with Cache Size $2500 \mathrm{MB}$

As seen in Figures 23 and 24, as the cache sizes get larger, EXD-AHP always offers the lowest amount of network traffic resulting from misses. At the larger cache sizes, PBPS + Hierarchy outperforms PBPS alone.

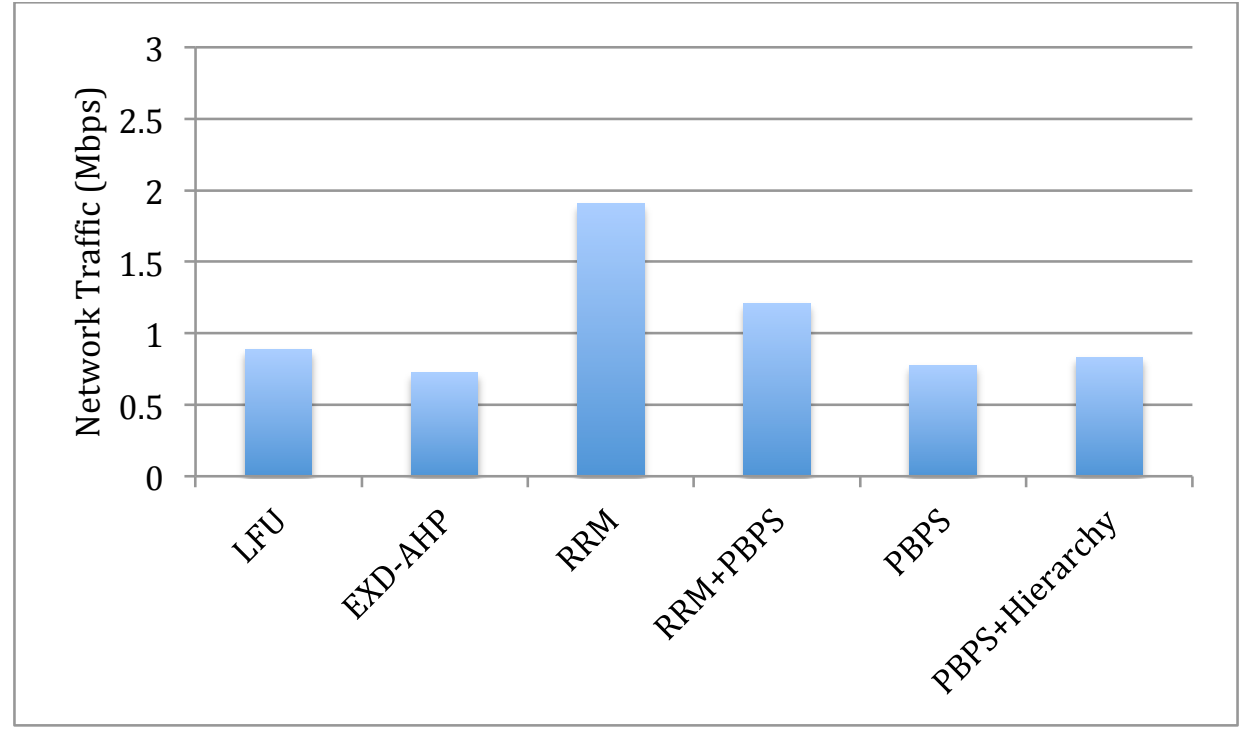

Figure 23. Network Traffic with Cache Size 3750 MB 


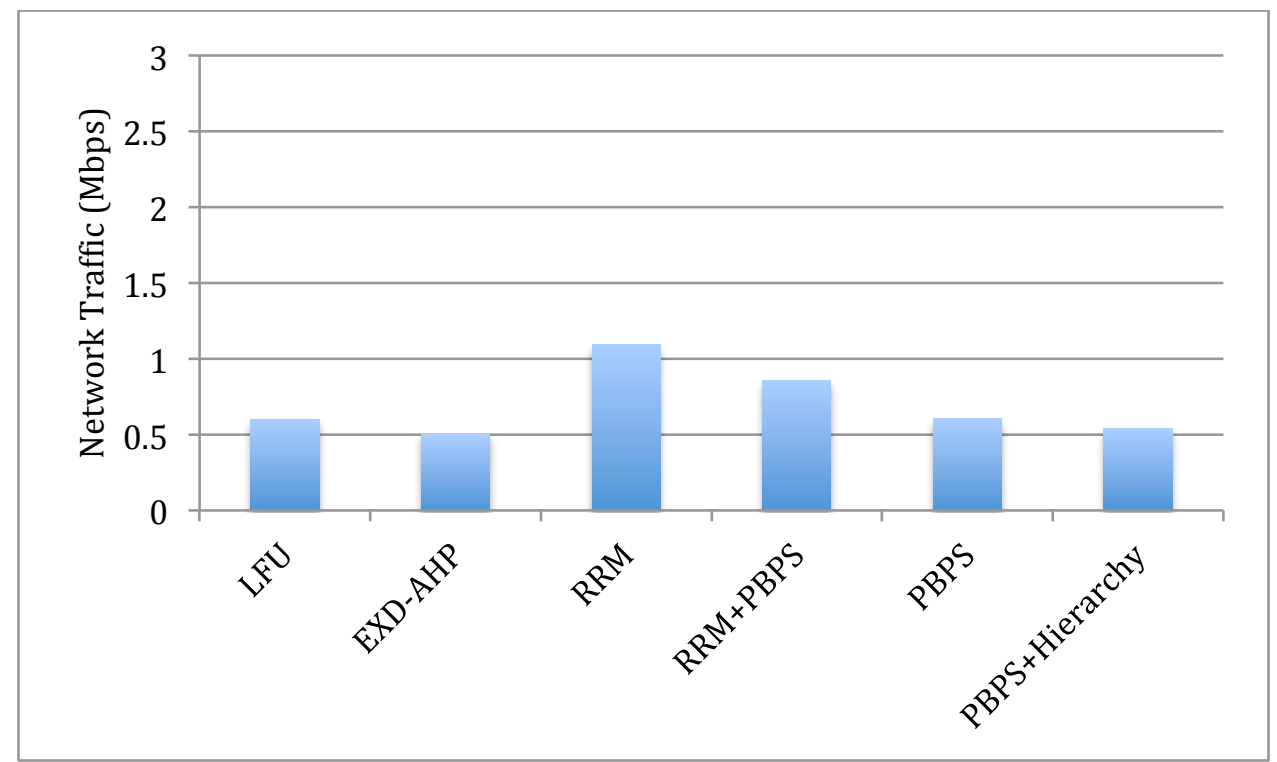

Figure 24. Network Traffic with Cache Size 5000 MB

As seen in every metric, RRM alone is not very well suited for the 5G C-RAN architecture type and service level differentiation, but can certainly be used once implemented in conjunction with the PBPS enhancement.

With any of the performance metrics, it is important to note that the cache size will always have an effect on the performance of the caching scheme. The larger the cache size is, the greater the number of UECs it can hold, and ultimately the better the performance is. Great variation can be seen in terms of how the different algorithms operate on smaller caches. Because of this, a cellular network service provider has the option to choose which algorithm it would prefer to manage the cache, based on what kinds of service it promises to offer or what types of service demands are prioritized over others. For instance, a provider could deem more important the distribution of caching amongst the service 
levels, or perhaps simply assuring that higher service levels are satisfied before other lower service levels. Whatever the demands and priorities are for a particular cellular network, these results showcase the effects of using different caching schemes, and allows the provider many options in cache management suitable to network needs. 


\section{CONCLUSION}

The research conducted in this paper and through subsequent experiments and simulation aimed to look at different caching techniques for User Equipment Contexts (UECs) in the $5^{\text {th }}$ Generation Cloud-RAN architecture. In terms of hit rates, latency, and cloud writes, there were tradeoffs found between EXD-AHP and PBPS + Hierarchy. EXD-AHP consistently offered great service to the highest service levels, whereas PBPS + Hierarchy offered more competitive distributions of results amongst the four service levels. PBPS + Hierarchy may be preferred with smaller cache sizes, but EXD-AHP performs best with larger cache sizes. PBPS on its own certainly does offer acceptable results, but its combination with the hierarchy yields the greatest benefits.

The RRM algorithm, which generally is not built to properly support service levels in Cloud-RAN, can be enhanced with PBPS for better performance in every sense and metric. The enhanced version of the original RRM + PBPS is now a feasible option for use in 5G C-RAN if the cellular network provider wanted to utilize it.

Results obtained from the Least Frequently Used algorithm, even in its best case scenario, in the 5G C-RAN Context consistently demonstrate the need for newer algorithms which can better serve this cellular architecture.

Future work and research can be done in finding better and improved caching techniques, which both offer faster service and can differentiate and provide appropriate treatment of service levels among users. Research could continue in the use of hierarchical or partitioned caches, and possibly how to dynamically adjust partitions or levels of the cache to best serve users and service levels. Another aspect that could be 
researched in the future is how to get appropriate hit rates in cache management systems with the lowest possible computational effort. Outside of caching, other areas can also be researched to improve service in 5G C-RAN, including the internal handlings of cloud data center resources in order to maximize utilization and lower costs in other areas, such as energy consumption, and maintaining their efficiency. 


\section{REFERENCES}

[1] Anand, K. and Barua, R. "Instruction-Locking for Improving Embedded Systems Performance," ACM Transactions on Embedded Computing Systems, Vol. 14, No. 3, Article 53, April 2015.

[2] Baek, S., Cho, S., and Choi, J. "Don't Make Cache Too Complex: A Simple Probability-Based Cache Management Scheme for SSDs," Public Library of Science ONE, 12(3): e0174375, March 2017.

[3] Calheiros, R. N. et al. "CloudSim: A ToolKit for Modeling and Simulation of Cloud Computing Environments and Evaluation of Resource Provisioning Algorithms," Software: Practice and Experience, 41.1, 23 - 50, 2011.

[4] Chow, F. "Why Virtualization is Essential for 5G," Silicon Valley 5G Summit, 2015.

[5] Fan, C. Zhang, Y. J., and Yuan, X. "Advances and Challenges Towards a Scalable Cloud Radio Access Network," IEEE Communications Magazine, Vol. 54, Issue 6, June 2016.

[6] Floratou, A., et al. "Adaptive Caching in Big SQL using the HDFS Cache," Proceedings of the Seventh ACM Symposium on Cloud Computing (SoCC '16), New York, NY, USA, 321-333, 2016.

[7] Gaur, J., Alameldeen, A. R., and Subramoney, S. "Base-Victim Compression: An Opportunistic Cache Compression Architecture," 2016 ACM/IEEE 43 ${ }^{\text {rd }}$ Annual International Symposium on Computer Architecture (ISCA), Seoul, South Korea, June 2016. 
[8] Huang, X., Zhao, Z. and Zhang, H. "Latency Analysis of Cooperative Caching with Multicast for 5G Wireless Networks," 2016 IEEE/ACM $9^{\text {th }}$ International Conference on Utility and Cloud Computing (UCC), Shanghai, China, Dec. 2016.

[9] Karneyenka, U. Mohta, K. and Moh, M. "Location and Mobility Aware Resource Management for 5G Cloud Radio Access Networks,” 2017 International Conference on High Performance Computing and Simulatin (HPCS), Genoa, Italy, July 2017.

[10] Kumar, B. A. and Rao, P. T. "Overview of Advances in Communication Technologies," $201513^{\text {th }}$ International Conference on Electromagnetic Interference and Compatibility (INCEMIC), Visakhpatnam, India, July 2015.

[11] Reguri, V. R., Kogatam, S. and Moh, M. "Energy Efficient Traffic-Aware Virtual Machine Migration in Green Cloud Data Centers," Proceedings of the Second IEEE International Conference on High Performance and Smart Computing, New York, April 2016.

[12] Tsai, C. and Moh, M. “Abstract: Cache Management and Load Balancing for 5G Cloud Radio Access Networks," ACM Symposium on Cloud Computing, Santa Clara, USA, Sept 2017.

[13] Tsai, C. and Moh, M. "Cache Management for 5G Cloud Radio Access Networks," Proceedings of ACM International Conference on Ubiquitous Information Management and Communication, to be held in Langkawi, Malaysia, January 2018.

[14] Wang, M. and Li, Z. "A Spatial and Temporal Locality-Aware Adaptive Cache Design with Netowrk Optimization for Tiled Many-Core Architectures," IEEE 
Transactions on Very Large Scale Integration (VLSI) Systems, Volume 25, Issue 9, Sept. 2017.

[15] Wang, X., et al. "Cache in the Air: Exploiting Content Caching and Delivery Techniques for 5G Systems," IEEE Communications Magazine, Vol. 52, Issue 2, February 2014.

[16] Ye, F., et al. "A Regional Popularity-Aware Cache Replacement Algorithm to Improve the Performance and Lifetime of SSD-Based Disk Cache," 2015 IEEE International Conference on Networking, Architecture and Storage (NAS), Boston, MA, USA, Aug. 2015.

[17] Zaidenberg, N., Gavish, L., and Meir, Y. "New Caching Algorithms Performance Evaluation,” International Symposium on Performance Evaluation of Computer and Telecommunication Systems (SPECTS), Chicago, IL, USA, July 2015.

[18] "5G Network Architecture: A High Level Perspective," Huawei Technologies Co., Ltd. Whitepaper, 2016. 